\title{
Design principles for digital value co-creation networks: a service-dominant logic perspective
}

\author{
Michael Blaschke ${ }^{1,2}$ (D) Uwe Riss ${ }^{3,4} \cdot$ Kazem Haki $^{2} \cdot$ Stephan Aier $^{2}$ \\ Received: 15 September 2017 / Accepted: 8 July 2019 / Published online: 19 August 2019 \\ (C) The Author(s) 2019
}

\begin{abstract}
Information systems (IS) increasingly expand actor-to-actor networks beyond their temporal, organizational, and spatial boundaries. In such networks and through digital technology, IS enable distributed economic and social actors to not only exchange but also integrate their resources in materializing value co-creation processes. To account for such IS-enabled value co-creation processes in multi-actor settings, this research gives rise to the phenomenon of digital value co-creation networks (DVNs). In designing DVNs, it is not only necessary to consider underpinning value co-creation processes, but also the characteristics of the business environments in which DVNs evolve. To this end, our study guides the design of DVNs through employing servicedominant logic, a theoretical lens that conceptualizes value co-creation as well as business environments. Through an iterative research process, this study derives design requirements and design principles for DVNs, and eventually discusses how these design principles can be illustrated by expository design features for DVNs.
\end{abstract}

Keywords Digital value co-creation networks (DVNs) - Design requirements · Design principles · Design features · Service-dominant (S-D) logic · Design science research (DSR)

JEL classification $\mathrm{O} 3$

This article is part of the Topical Collection on Design Science Research in the Networked Economy

\section{Responsible Editor: Jan vom Brocke}

Michael Blaschke

michael.blaschke@unisg.ch

Uwe Riss

uwe.riss@fhsg.ch

Kazem Haki

kazem.haki@unisg.ch

Stephan Aier

stephan.aier@unisg.ch

1 Information Systems and Innovation Faculty Research Group, London School of Economics and Political Science, 54 Lincoln's Inn Fields, London WC2A 3LJ, United Kingdom

2 Institute of Information Management, University of St. Gallen, Müller-Friedberg-Str. 8, 9000 St. Gallen, Switzerland

3 Institute of Information and Process Management, University of Applied Sciences, Rosenbergstrasse 59, 9000 St. Gallen, Switzerland

4 School of Humanities, University of Hertfordshire, Hatfield, Hertfordshire AL10 9AB, United Kingdom

\section{Introduction}

"Future work may consider how individuals in complex multi-actor value networks perceive value through use or experience after exchanging and integrating resources by means of ICT. [...] ICTs can be used to transform the structure of value co-creation processes from co-located contexts into dynamic, distributed, and technology-enabled ones" (Breidbach and Maglio 2016, p. 83).

Marketing research apprises scholars in different disciplines of a paradigmatic reorientation from traditional goods-dominant (G-D) to a service-dominant (S-D) logic that reconceptualizes the notion of economic exchange (Vargo and Lusch 2004, 2008, 2016, 2017). S-D logic shifts the focus of economic exchange from value creation in a single organization to a broader network of social and economic actors (network-centric focus) (Lusch and Nambisan 2015). Moreover, S-D logic underscores that tangible goods are no longer the 
sole object of exchange, but also associated or stand-alone intangible offerings in which the extent of information content is high (information-centric focus) (Lusch and Nambisan 2015). Eventually, S-D logic sheds light on a shift in the outcome of economic exchange, from features and attributes of exchanged goods to the value that is $\mathrm{co}$-created during the use of exchanged goods (value-centric focus) (Lusch and Nambisan 2015). Thus, it is pivotal to S-D logic that value is determined by the quality of a value-in-use experience and not just by the quality of goods'value-in-exchange (Macdonald et al. 2016; Prahalad and Ramaswamy 2004). For instance, Hilti, a global market leader for professional drilling and mounting technologies, has started embracing SD logic with a first step of selling drilling equipment utilization (value-in-use, S-D logic) instead of selling drilling equipment (value-in-exchange, G-D logic) (vom Brocke et al. 2017). This reorientation in Hilti's economic exchange reflects that value only unfolds during the time of use in value co-creation processes between social and economic actors that reciprocally integrate resources in a business network (Grönroos 2011; Grönroos and Ravald 2011; Payne et al. 2008).

Information systems (IS) play an increasingly dominant role in such value co-creation processes (Lusch and Nambisan 2015) in that they expand actor-to-actor networks beyond their temporal, organizational, and spatial boundaries. Thus, economic and social actors increasingly exchange and integrate resources in multi-actor settings facilitated by digital technology (Breidbach and Maglio 2016; Davis et al. 2011). Predominantly, IS research focuses on the emergence of globallyconnected digital infrastructures as socio-technical systems (Henfridsson and Bygstad 2013; Tilson et al. 2010). To account for such IS-enabled value cocreation processes in multi-actor settings, this research gives rise to the phenomenon of digital value cocreation networks (DVNs) as the investigated IS phenomenon in this study. We use the notion of digital value co-creation network to denote a specific class of service ecosystems (Böhmann et al. 2014) - ones inextricably intertwined with and facilitated by a digital infrastructure, aimed at digital service through dynamic value co-creation, and constituted of multi-actor settings contingent on a given service beneficiary's (e.g., end user organization or consumer) needs. Apple (iOS), Alphabet (Android), Microsoft (Azure), or Amazon.com represent prime examples of DVN orchestrators-the design principles' targeted users. These organizations (re)form DVNs with various platform-augmenting third parties and subcontractors to deliver a digital service to a given service beneficiary. Here, we designate DVNs as complex, socio-technical service ecosystems to configure emergent, networked, and IS-enabled value co- creation processes resulting in digital service (Breidbach and Maglio 2016; Lusch and Nambisan 2015).

In this way, DVNs extend the scale and scope of value co-creation processes toward complex configurations of organizational (e.g., business processes) and technological (e.g., software, hardware) elements (Böhmann et al. 2014). The rates and patterns of change in these sociotechnical elements testify to the inherent complexity in designing DVNs (Briscoe et al. 2012). Against this backdrop, DVNs' survival under such complex design conditions is contingent on a design that delicately adjusts DVNs' defining characteristics in which DVNs are built. In the face of the outlined complex design conditions, this study passes from theoretical consideration to actionable design guidance by answering the following research question: What are the principles for guiding the design of DVNs that account for the requirements of value cocreation? Owing to S-D logic's distinctive and penetrative conceptualization on how value is co-created in an actorto-actor network, we employ S-D logic as a kernel theory (Vargo and Lusch 2004, 2008, 2016). In that regard, S-D logic guides our derivation of DVN design requirements based on which we derive the respective DVN design principles. Ultimately, for an illustrative DVN instance, we present expository DVN design features that illustrate specific technical ways to instantiate the proposed design principles.

The remainder of this paper is structured as follows. Research Background presents the DVN concept and S$\mathrm{D}$ logic as this study's theoretical background. Research Method describes our design science research process and the employed research methods. Results presents the resultant DVN design requirements and principles. Illustration and Evaluation illustrates and evaluates the proposed design principles. Discussion and Conclusion discusses the derived tripartite organizing structure of interrelated DVN requirements, principles, and features and provides concluding remarks.

\section{Research background}

Drawing on and integrating into extant value co-creation and S-D logic research, we give rise to the phenomenon of DVNs, briefly synthesize DVNs' conceptual constituents (i.e., digital infrastructure, value co-creation, and actor-to-actor networks), and introduce S-D logic as employed kernel theory.

\section{Digital value co-creation networks}

Still, little light has been shed on how actors engage in contexts of dyadic and physical resource integration (Breidbach 
and Maglio 2016), let alone in multilateral and digital resource integration (Beirão et al. 2017; Lusch and Nambisan 2015). The notion of DVN - the central phenomenon investigated in this research - is characterized by a "heterogeneous and dynamic pool of actors and tools that need to be dynamically identified and mobilized for effective cognitive and social translations across a diverse set of actors in the absence of hierarchical control and presence of high levels of knowledge heterogeneity" (Lyytinen et al. 2016, p. 59). Service research, therefore, has moved from the traditional view of dyadic oneto-one service encounters to a more encompassing view of multilateral many-to-many service encounters within service ecosystems (Barile et al. 2016; Chandler and Lusch 2015; Maglio et al. 2009). Such service ecosystems enable networks of actors to co-create value (Barile et al. 2016).

DVNs, in turn, are a type of service ecosystem in which ISenabled networks of actors co-create digital service. Therefore, DVN design is depends on a fine-tuned, aligned configuration of three defining characteristics. DVNs represent service ecosystems that are

(1) inextricably intertwined with and facilitated by a digital infrastructure (i.e., socio-technical) —including digital technology, data, and physical artifacts (e.g., hardware) (Böhmann et al. 2014),

(2) aimed at digital service that results from value cocreation processes among DVN orchestrators, third parties, and service beneficiaries (i.e., dynamic), and

(3) constituted of networks of actors that (re)form to (re)conform to service beneficiaries (i.e., complex).

Prominent DVN orchestrators - the DVN design principles' targeted users - such as Apple (iOS), Alphabet (Android), Microsoft (Azure, Windows), or Amazon.com operate under such premises by building up digital infrastructures, upon which their respective DVNs dynamically (re)form to cater to the needs of a given service beneficiary (e.g., end-user organization or consumer). Accounting for such a fine-tuned configuration of these three characteristics, we ground this research on digital infrastructure, value co-creation, and networks of actors.

Digital infrastructure As DVNs are devised in infrastructural arrangements of digital technology, we take an infrastructure view on IT and IS in this research (Henfridsson and Bygstad 2013). We rely on this view as globally distributed actor-toactor networks are created and cultivated on top of digital infrastructures (DIs) - here defined as computing and network resources that allow distributed actors to facilitate their resource exchange (Constantinides et al. 2018). The Internet, data centres, open standards (e.g., IEEE 802.11 and USB), and consumer devices (e.g., smartphones and tablets) are prime DI examples. DIs, therefore, are distinct from other types of infrastructures because of their ability to collect, store, and make digital data available across several systems and devices (Henfridsson and Bygstad 2013). This view is in line with the more general notion that digitalization applies "digitizing techniques to broader social and institutional contexts that render digital technologies infrastructural" (Tilson et al. 2010 , p. 749). In turn, such infrastructural rendering of digital technologies in networks of actors has led to the evolution of DIs (Hanseth and Lyytinen 2010; Henfridsson and Bygstad 2013; Tilson et al. 2010). Extant IS research reports on the Internet of Things (Papert and Pflaum 2017), big data services (Alt and Zimmermann 2017; Loebbecke and Picot 2015), or social media platforms (Baumol et al. 2016) as prominent DI instances through which actors integrate resources in a novel way. These studies observe a shift from individual organizations' IT infrastructures to networks of inter-organizational information infrastructures (Ciborra 2000; Tilson et al. 2010), and from stand-alone IS to interconnected IS collectives (Henfridsson and Bygstad 2013, p. 908). DI captures "the technological and human components, networks, systems, and processes" that contribute to the functioning of a DVN (Henfridsson and Bygstad 2013, p. 908). DIs' unique properties are fundamental in understanding value co-creation processes in multi-actor constellations (Tilson et al. 2010, p. 749).

Value co-creation While the concept of value co-creation has been discussed for more than a decade (e.g., Galvagno and Dalli 2014; Ranjan and Read 2016), S-D logic sophisticates this concept through establishing a holistic, unified, and precise theoretical foundation as a distinctive, yet complementary, perspective on extant debates (Vargo and Lusch 2017; Vargo et al. 2010). From an S-D logic vantage point, value co-creation is "the processes and activities that underlie resource integration and incorporate different actor roles in the service ecosystem" (Lusch and Nambisan 2015, p. 162). Value co-creation underscores that all actors integrate resources and engage in service exchange - all in the process of synergistically and reciprocally co-creating value (Vargo and Lusch 2016 , p. 3). In this process, actors integrate resources through service exchange, configured by institutional arrangements through which service ecosystems endogenously emerge (Vargo and Lusch 2016, p. 3).

Networks of actors Value co-creation increasingly manifests in configurations of multilateral offeror-to-beneficiary constellations comprising multiple actors (Akaka et al. 2012; Barile et al. 2016; Beirão et al. 2017). This is reflected in SD logic's network-centric focus. Such a network approach to value co-creation emphasizes the larger constellations within which multiple, varied, and interdependent actors (re)form to serve a given service beneficiary. Such networks are characterized as "spontaneously sensing and responding spatial and 
temporal structures of largely loosely coupled, valueproposing social and economic actors interacting through institutions, technology, and language to (1) co-produce service offerings, (2) engage in mutual service provision, and (3) cocreate value" (Vargo and Lusch 2011, p. 185). Thus, in designing DVNs, it is necessary to consider the characteristics of value co-creation as well as actor-to-actor networks in which DVNs evolve. To this end, we adopt S-D logic as kernel theory to conceptualize both value co-creation and business environments.

\section{Theoretical Foundation: A service-dominant logic perspective}

We propose S-D logic as the main theoretical lens for this study to conceptualize both value co-creation as well as emerging networked business environments. S-D logic is rooted in marketing research, where it gained momentum since its inception by the landmark study of Vargo and Lusch (2004), followed by further amendments (Vargo and Lusch 2008, 2016). We synthesis knowledge of S-D logic on four levels to differentiate and reflect these levels' descriptive and prescriptive nature (see Fig. 1).

S-D logic has been introduced through descriptive theoretical assumptions, which are formulated as metatheoretical foundations of S-D logic (Level I) (Lusch and Nambisan 2015; Lusch et al. 2010). Subsequently, scholars captured these foundations in a set of S-D logic's foundational premises to explicate S-D logic's worldview (Level II) (Vargo and Lusch 2004, 2008, 2016). Later, scholars started elaborating the managerial implications of S-D logic's theoretical foundations in real-world practices. This endeavor resulted in a set of derivative propositions that inform practitioners about competition in an S-D logic orientation (Level III) (Lusch et al. 2007). Levels I to III are offered by seminal S-D logic literature. They provide descriptive knowledge to explicate S-D logic with an increasing degree of applicability in practice. Drawing on these three levels, we position our study as one step further in translating S-D logic's descriptive basis into prescriptive means in our phenomenon of interest. As such, the central outcome of this design science research (DSR) is prescriptive knowledge in the form of design requirements and design principles for DVNs (Level IV).

Emphasizing the move from descriptive to prescriptive knowledge, Fig. 1 summarizes these levels, each of which is briefly explained below. Further building on the seminal S-D logic studies, Table 1 shows the relation between constituents of Level I, II, and III.

Meta-theoretical foundations (level I) On a meta-theoretical level, S-D logic is grounded in and derived from four metatheoretical foundations, namely actor-to-actor networks, resource liquefaction, resource density, and resource integration (Lusch and Nambisan 2015, p. 164). Actor-to-actor-networks emphasize a shift from one-way processes of value exchange in traditional supply chains (i.e., neoclassical industrial

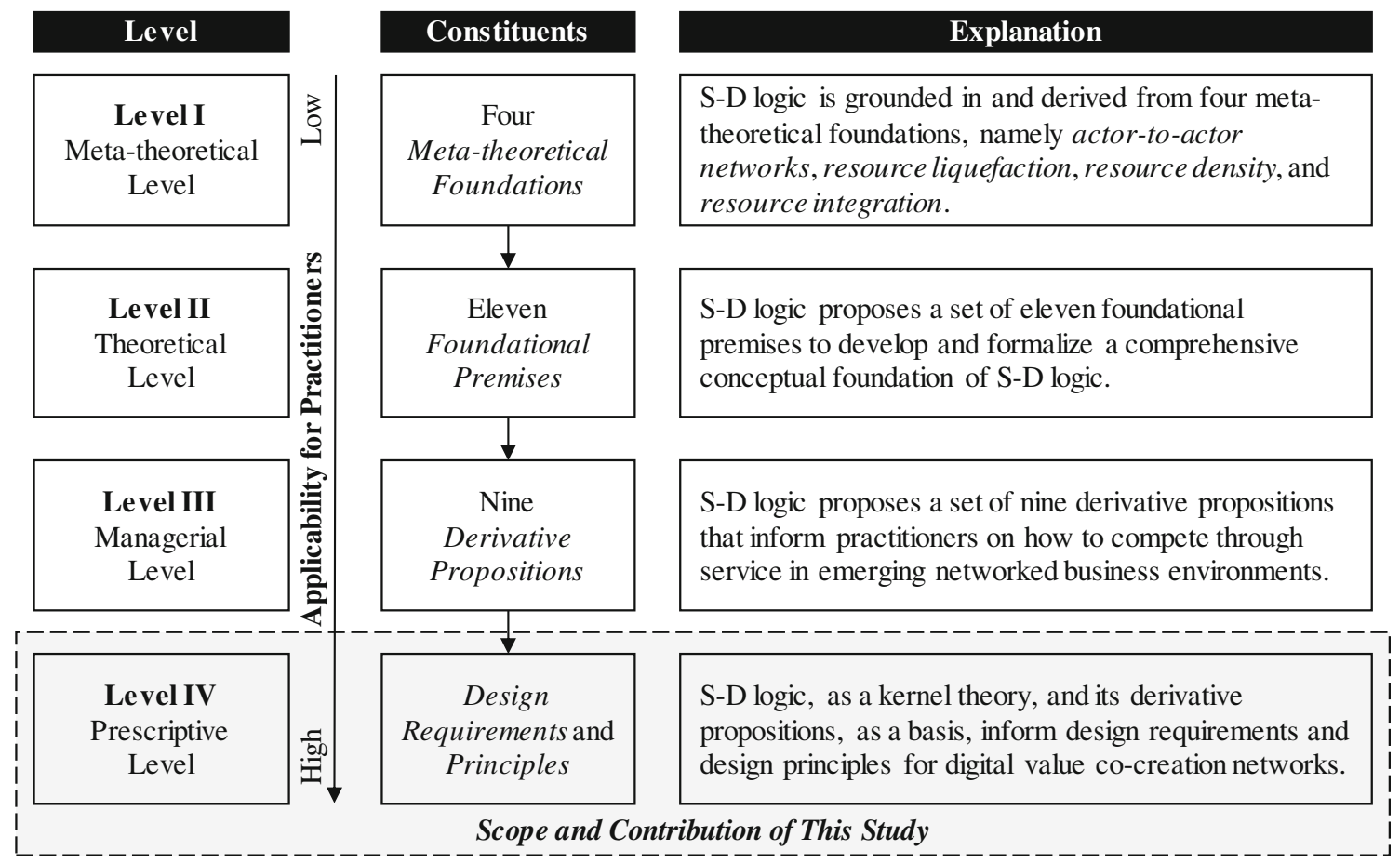

Fig. 1 Service-dominant logic: from descriptive to prescriptive knowledge 
Table 1 Service-Dominant Logic: The Relations between Meta-Theoretical Foundations, Foundational Premises, and Derivative Propositions (with relations in brackets)

Meta-Theoretical Foundations (MFs) (Lusch and Nambisan 2015)
Foundational Premises (FPs) (Vargo and Lusch 2004, 2008, 2016) in Association to MFs (Lusch and Nambisan 2015)

FP1. Service is the fundamental basis of exchange. (MF1)

FP2. Indirect exchange masks the fundamental basis of exchange. (MF1, MF3)
MF2 (Resource Liquefaction). S-D logic draws on the decoupling of information from its related physical form or device.

MF3 (Resource Density). S-D logic draws on an effective and efficient mobilization of contextually relevant knowledge.

FP3. Goods are distribution mechanisms for service provision. (MF3)

FP4. Operant resources are the fundamental source of strategic benefit. (MF2)

FP5. All economies are service economies. (MF1)

FP6. Value is co-created by multiple actors, always including the beneficiary. ( $M F 1$, MF4)

MF4 (Resource Integration). S-D logic draws on the view that all social and economic actors are resource integrators.
FP7. Actors cannot deliver value but can participate in the creation and offering of value propositions. ( $M F 1)$

FP8. A service-centered view is inherently beneficiary oriented and relational. (MF4)

FP9. All social and economic actors are resource integrators. (MF1, MF4)

FP10. Value is always uniquely and phenomenologically determined by the beneficiary. (MF4)

FP11. Value co-creation is coordinated through actor-generated institutions and institutional arrangements. (MF1, MF4)
Derivative Propositions (Lusch et al. 2007) in

Association to FPs (Vargo and Lusch 2004, 2008, 2016)

DP1. Competitive advantage is a function of how one firm applies its operant resources to meet the needs of the customer relative to how another firm applies its operant resources. (FP1, FP4)

DP2. Collaborative competence is a primary determinant of a firm's acquiring the knowledge for competitive advantage. (FP4, FP9)

DP3. The continued ascendance of IT with associated decrease in communication and computation costs, provides firms opportunities for increased competitive advantage through innovative collaboration. (FP6, FP8)

DP4. Firms gain competitive advantage by engaging customers and value network partners in co-creation and co-production activities. ( $F P 6, F P 9$ )

DP5. Understanding how the customer uniquely integrates and experiences service-related resources (both private and public) is a source of competitive advantage through innovation. (FP6, FP8, FP9)

DP6. Providing service co-production opportunities and resources consistent with the customer's desired level of involvement leads to improved competitive advantage through enhanced customer experience. (FP6, FP8, FP9)

DP7. Firms can compete more effectively through the adoption of collaboratively developed, risk-based pricing value propositions. (FP6, FP7)

DP8. The value network member that is the prime integrator is in a stronger competitive position. The retailer is generally in the best position to become the prime integrator. (FP1, FP4, FP9)

DP9. Firms that treat their employees as operant resources will be able to develop more innovative knowledge and skills and thus gain competitive advantage. (FP4) perspective) to collaborative processes of value co-creation in service ecosystems (i.e., network-centric perspective). Resource liquefaction underscores a shift from information coupled to its related physical matter to digitized, decoupled, and more useful information easier to share with others. Resource density emphasizes a shift from the mobilization of resources for integration (i.e., low resource presence) at a given time and place to the mobilization of a combination of contextually relevant resources for a situation (i.e., maximum resource density). Resource integration, eventually, underscores a shift from the production of fixed-asset goods to the integration of specialized resources into complex services, demanded by service beneficiaries in a specific context.

Foundational premises (level II) On a theoretical level, Vargo and Lusch (2004) proposed - and further amended (Vargo and Lusch 2008, 2016) — a set of foundational premises (FPs) for S-D logic in distinction to a G-D logic. This effort has culminated in eleven FPs (Vargo and Lusch 2016), which explicate the ontological basis of S-D logic and which are related to S-D 
logic's meta-theoretical foundations (see Table 1). For instance, FP6 and FP9 are derived from the meta-theoretical foundation resource integration. As such, value is co-created by multiple actors, always including the beneficiary (FP6) and all social and economic actors are resource integrators (FP9) (Vargo and Lusch 2016). In promoting FPs, S-D logic reconceptualizes service (the process of applying specialized competencies for the benefit of one another), exchange (not the exchange of outputs but the exchange of the performance of specialized activities), value (occurs when the offering is useful to service beneficiary), and resource (anything an actor can draw on for support) (Lusch and Nambisan 2015).

Regarding resource, S-D logic distinguishes operand and operant resources. Operand resources refer to tangible, static, and passive components of goods that actors employ to obtain support (Vargo and Lusch 2004). In S-D logic, they are seen as "vehicles for service provision, rather than primary to exchange and value creation" (Pels and Vargo 2009, p. 374). In contrast, operant resources refer to intangible, dynamic, and active resources (e.g., human knowledge, skill, and experience) that act on other resources (Vargo and Lusch 2004). In S-D logic, operan $t$ resources have a pivotal role since they are seen as "the fundamental source of competitive advantage "(Vargo and Lusch 2008, p. 7). S-D logic perceives IT artifacts as both facilitator of service exchange among actors (operan $d$ ) and trigger of value co-creation activities and processes (operant) (Lusch and Nambisan 2015).

Derivative propositions (level III) On a managerial level, building on the foundational premises (FPs) of S-D logic (Vargo and Lusch 2004), Lusch et al. (2007) derived nine propositions as practical implications of the FPs to inform practitioners about competing through service (see Table 1). The overall theme of these derivative propositions is innovate and compete through service thinking. They start from the premise that in order to "survive and prosper in a networked economy, the organization must learn how to be a vital and sustaining part of the value network" (Lusch et al. 2010, p. 21). In the context of this study, we rely on these derivative propositions as a starting point in deriving DVN design requirements.

Design knowledge (level IV) In this study, we propose a new level for the development of artifacts that help practitioners exercising the promoted service thinking of S-D logic. We draw on Lusch et al.'s (2007) generic derivative propositions to derive specific design requirements and design principles that help organizations build DVNs and, thus, incorporate S-D logic. While the derivative propositions seek to outline the relationship between service thinking (S-D logic's theoretical foundations) and gaining competitive advantage, the targeted design requirements and design principles seek to guide the design of DVNs informed by service thinking. Passing from theoretical consideration to practical design guidance, this study is in pursuit of the principles for guiding the design of DVNs that account for the requirements of value cocreation and emerging business environments.

\section{Research method}

In this section we present our adoption of Sonnenberg and vom Brocke's (2012, p. 392) cyclic DSR process and its extension by Abraham et al. (2014). This staged research process guides our systematic identification of design requirements and design principles for DVNs. We employ this process as it (1) incorporates a design-evaluateconstruct-evaluate pattern, and as it (2) includes the DSR activities problem identification, design, construction, and use followed by four distinct corresponding evaluation activities referred to as Evall to Eval4. Ensuring multiple evaluation episodes throughout a single iteration of a DSR process, these four evaluation activities allow for a continuous assessment of the progress achieved in devising the targeted design principles (Abraham et al. 2014; Sonnenberg and vom Brocke 2012, p. 390). Table 2 summarizes the applied build and evaluation activities to devise the targeted design principles.

Adapting the four evaluation episodes (see Table 2), we account for three principles of systematic evaluation in DSR: (1) applying ex-ante and ex-post evaluation modes, (2) documenting cumulative prescriptive knowledge, and (3) continuously assessing the progress achieved in a DSR process. To design and conduct each of these episodes rigorously, we adopt the Framework for Evaluation in Design Science (FEDS) (adapted from Venable et al. 2014) (see Table 3). We applied FEDS to design and conduct EVAL1- EVAL4. Table 3 synthesizes our adoption of FEDS.

As an evaluation strategy (FEDS Step I.), we adopt the evaluation strategy human risk \& effectiveness (Venable et al. 2014, p. 81) as it is effective if, as true in our DSR project, (1) the major design risk is social and user-oriented, and if (2) it is relatively cheap to evaluate the design principles with real users in their real context. Alternatives would be quick \& simple, purely technical artifact, or technical risk evaluations.

As evaluation goals (FEDS Step II.), episodes EVAL1 and EVAL2 aim to reduce the uncertainties in (1) solving an irrelevant and/or solved problem and (2) specifying ineffective design requirements. Episodes EVAL3 and EVAL4 aim to increase rigor in establishing that the design principles' utility will continue in the long run. While FEDS Step I and Step II are outlined above, Table 3 summarizes FEDS Step III 
Table 2 The Applied Build and Evaluation Activities of the Cyclic DSR Process in our Design Principles Development (adapted from Abraham et al. 2014; Sonnenberg and vom Brocke 2012)

\begin{tabular}{|c|c|c|c|c|}
\hline Activity & Purpose of Activity & Applied Method & Output & Context \\
\hline $\begin{array}{l}\text { 1.1. PROBLEM } \\
\text { IDENTIFICAT- } \\
\text { ION }\end{array}$ & Selecting and formulating a problem statement & $\begin{array}{l}\text { Review of } \\
\text { Practitioner } \\
\text { Initiative }\end{array}$ & $\begin{array}{l}\text { Justified Problem Statement: } \\
\text { Practitioners lack actionable guidance to } \\
\text { efficiently and effectively build DVN }\end{array}$ & \multirow[t]{6}{*}{$\begin{array}{l}\text { R\&D Project at } \\
\text { Alpha (see } \\
\text { Table 4) }\end{array}$} \\
\hline 1.2. EVAL1 & Ensuring that the stated problem is meaningful & $\begin{array}{l}\text { Literature } \\
\text { Review, Focus } \\
\text { Group }\end{array}$ & designs. & \\
\hline 2.1. DESIGN & $\begin{array}{l}\text { Formulating design requirements for the stated } \\
\text { problem }\end{array}$ & $\begin{array}{l}\text { Literature } \\
\text { Review, } \\
\text { Logical } \\
\text { Reasoning }\end{array}$ & \multirow[t]{2}{*}{$\begin{array}{l}\text { Validated Design Requirements: } \\
\text { Employing S-D logic's nine derivative } \\
\text { propositions to identify DVN design } \\
\text { requirements }\end{array}$} & \\
\hline 2.2. EVAL2 & $\begin{array}{l}\text { Ensuring that the design requirements are } \\
\text { meaningful }\end{array}$ & $\begin{array}{l}\text { Logical } \\
\text { Reasoning, } \\
\text { Demonstration }\end{array}$ & & \\
\hline $\begin{array}{l}\text { 3.1. } \\
\text { CONSTRUCTI- } \\
\text { ON }\end{array}$ & Prototypically formulating the design principles & $\begin{array}{l}\text { Expert } \\
\text { Workshop, } \\
\text { Logical } \\
\text { Reasoning }\end{array}$ & \multirow{2}{*}{$\begin{array}{l}\text { Validated Design Principles in an } \\
\text { Artificial Setting: } \\
\text { Validating prototypically formulated } \\
\text { design principles against design } \\
\text { requirements }\end{array}$} & \\
\hline 3.2. EVAL3 & $\begin{array}{l}\text { Ensuring that design principles meet the design } \\
\text { requirements }\end{array}$ & Expert Interview & & \\
\hline 4.1. USE & $\begin{array}{l}\text { Fully formulating and using the design } \\
\text { principles in practice }\end{array}$ & $\begin{array}{l}\text { Design } \\
\text { Workshop } \\
\text { Observation }\end{array}$ & $\begin{array}{l}\text { Partially Validated Design Principles in a } \\
\quad \text { Naturalistic Setting: } \\
\text { Preliminarily validating fully formulated }\end{array}$ & $\begin{array}{l}\text { DVN Design } \\
\text { Workshop at Beta } \\
\text { (see Table 4) }\end{array}$ \\
\hline $\begin{array}{l}\text { 4.2. EVAL4 } \\
\text { (preliminary) }\end{array}$ & $\begin{array}{l}\text { Ensuring that the design principles are useful } \\
\text { and that principles-informed DVN designs } \\
\text { are effective }\end{array}$ & $\begin{array}{l}\text { Design } \\
\text { Workshop } \\
\text { Analysis }\end{array}$ & design principles with real users & \\
\hline
\end{tabular}

(evaluation criteria), FEDS Step IV (applied methods), and evaluation outputs.

We have conducted this research within three purposefully chosen contexts (see Table 4). First, for deriving the design principles (activities 1.1. PROBLEM IDENTIFICATION3.2. EVAL3), we analyzed an R\&D project at Alpha (a pseudonym), a multinational enterprise software vendor, investigating the systematic design of DVNs (see section Problem Identification and Eval1). Second, for illustrating the design principles, we analyze Alpha's DVN (see Appendix 1). Third, for naturalistically evaluating the design principles (see section Use and Eval4), we organize a DVN design workshop at Beta (a pseudonym), a large steel producer (see Appendix 2). Alpha is suitable organization for deriving (through its DVN-building R\&D project) and illustrating (through highlighting its DVN's expository design features) the design principles as it has operated a thriving DVN since 2012. Conversely, Beta is a suitable organization for naturalistically evaluating them (through using the proposed design principles in Beta's DVN design workshop) as Beta has limited experience and demands actionable guidance in building DVN designs. Table 4 presents these three settings, relying on which we derive the design principles for DVNs from an $S-D$ logic perspective within the context of Alpha's DVN-building R\&D project.

\section{Problem identification and Eval1}

Alpha's R\&D project aimed at investigating the systematic design of DVNs in a co-innovation format with 20 senior executives of European multi-national enterprises. In deriving the targeted DVN design principles, we draw on the design knowledge accumulated during the period from 2015 to 2018 of Alpha's R\&D project, in which two of the co-authors were involved. The senior executives participated in Alpha's R\&D project to initiate new or improve existing DVN designs for their organizations. Alpha's R\&D project entailed consulting activities, workshops, and trainings, all of which were related to building DVN designs in the various contexts of this project's co-innovation partners. These activities revealed that the managers and designers at DVN orchestrators - the design principles' targeted userslack actionable guidance in building DVN designs. At the outset, Alpha's R\&D project drew upon established visual inquiry and business model design techniques (e.g., business model canvases (Wirtz et al. 2016)). It became clear that these techniques are limited in accounting for the practical and theoretical requirements of value co-creation and emerging networked business environments in building DVN designs. One reason is that these techniques do not sufficiently address the socio-technical, dynamic, and complex nature of DVNs. This lack can be attributed to the fact that these 
Table 3 The Applied Framework for Evaluation in Design Science (FEDS) in our Design Principles Evaluation (adapted from Venable et al. 2014)

\begin{tabular}{|c|c|c|c|}
\hline Activity & $\begin{array}{l}\text { Evaluation } \\
\text { Criteria }\end{array}$ & Applied Method & Output \\
\hline 1.2. EVAL1 & $\begin{array}{c}\text { Relevance, } \\
\text { novelty }\end{array}$ & $\begin{array}{l}\text { - Ex-ante, artificial evaluation via literature review }{ }^{\text {a }} \text { aims to validate problem } \\
\text { novelty. Two authors analyze } 30 \text { selected papers regarding the identified } \\
\text { problem, theoretical requirements, and solution components. } \\
\text { - Ex-ante, naturalistic evaluation via focus group aims to validate relevance. } \\
\text { One author follows an interview guideline in a one-hour interview with } \\
\text { four of Alpha's applied researchers. }\end{array}$ & $\begin{array}{l}\text { - Literature does not offer actionable DVN } \\
\text { design guidance. } \\
\text { - DVN designers lack and request } \\
\text { actionable DVN design guidance. }\end{array}$ \\
\hline 2.2. EVAL2 & $\begin{array}{l}\text { Feasibility, } \\
\text { complete- } \\
\text { ness }\end{array}$ & $\begin{array}{l}\text { - Ex-ante, artificial evaluation via logical reasoning aims to validate } \\
\text { completeness of S-D logic. The four authors review candidates for ef- } \\
\text { fective kernel theories that inform DVN design requirements. } \\
\text { - Ex-ante, natural evaluation via demonstration in a 90-min long working } \\
\text { meeting with two Alpha DVN experts and five executives aims to vali- } \\
\text { date S-D logic's nine derivative propositions to inform DVN design re- } \\
\text { quirements. }\end{array}$ & $\begin{array}{l}\text { - S-D logic's nine derivative propositions } \\
\text { serve to inform DVN design require- } \\
\text { ments. } \\
\text { - Design principles are effective for } \\
\text { guiding DVN design. }\end{array}$ \\
\hline 3.2. EVAL3 & $\begin{array}{l}\text { Applicability, } \\
\text { efficacy }\end{array}$ & $\begin{array}{l}\text { Ex-post, artificial evaluation via expert interview aims to validate the } \\
\text { prototypical design principles' applicability and efficacy. Two authors } \\
\text { follow a semi-structured guideline to interview the same five executives } \\
\text { (see Eval2). All authors analyze and discuss taken notes in a 90-miunte } \\
\text { long workshop to reflect learnings in the prototypical design principles. }\end{array}$ & $\begin{array}{l}\text { Prototypically formulated design } \\
\text { principles are applicable and } \\
\text { efficacious. }\end{array}$ \\
\hline $\begin{array}{l}\text { 4.2. EVAL4 } \\
\text { (prelimi- } \\
\text { nary) }\end{array}$ & $\begin{array}{l}\text { Usefulness, } \\
\text { effective- } \\
\text { ness }\end{array}$ & $\begin{array}{l}\text { Ex-post, naturalistic evaluation via a DVN design workshop observation at } \\
\text { Beta with nine primary participants aims to validate usefulness of design } \\
\text { principles and effectiveness of principles-informed DVN designs. }\end{array}$ & $\begin{array}{l}\text { Preliminarily validated design principles } \\
\text { with a case firm aiming at DVN designs }\end{array}$ \\
\hline
\end{tabular}

${ }^{a}$ We include studies on S-D logic from 11 marketing journals that are ranked (world) leading (tagged with *) by at least one of the ratings included in the 57th Harzing Journal Quality List (2016). We search in the Business Source Premier database employing the EBSCOhost search engine since S-D logic's inaugural year 2004 (Vargo and Lusch 2004). 30 selected papers carry "service-dominant", "service logic", or "dominant logic" in title, abstract, or keywords. In addition, we include studies on and/or using S-D logic that are published in the AIS basket-of-eight journals. This adds another 15 papers, most of which are part of the MISQ special issues on "Service Innovation in the Digital Age" (Barrett et al. 2015) and on "Co-creating IT Value" (Grover and Kohli 2012)

techniques mainly provide design guidance for traditional value creation in supply chains while they fail to deal with the dynamics of DVNs' elaborated network structures (PROBLEM IDENTIFICATION).

IS research focuses on (1) informing the study of IS phenomena through S-D Logic and value co-creation as theoretical lens, or on (2) realizing S-D logic and value co-creation through digital means (Haki et al. 2018). Regarding the first focus (lens to inform), S-D logic has become increasingly influential in theorizing various IS phenomena (e.g., Ceccagnoli et al. 2012; Grover and Kohli 2012; Sarker et al. 2012). For example, IS research not only conceptualizes service innovation (e.g., Barrett et al. 2015; Lusch and Nambisan 2015), but has also initiated investigations on service innovation (e.g., Nambisan 2013; Srivastava \& Shainesh 2015). Regarding the second focus (means to realize), research illustrates the realization of S-D logic and value co-creation through digital means (e.g., Ordanini and Pasini 2008; Yan et al. 2010). Such studies also discuss S-D logic's and value co-creation's practical implications on designing digital means (e.g., Böhmann et al., 2018). This second focus, however, is still a maturing discussion so that it lacks guidance on how to design digital means in enabling and realizing S-D logic and value co-creation. Therefore, due to its theoretical orientation, the S-D logic and IS literature lack guidance in building DVN designs (Akaka \& Vargo, 2014; Breidbach \& Maglio, 2016, p. 83). The identified problem-S-D logic offers no guidance in building DVN designs - hence is also academically relevant.

To validate whether this problem is meaningful for practitioners, we conducted a one-hour focus group with four Alpha employees that were applied researchers working on Alphas's R\&D project. Beyond their accessibility and supportiveness, we chose these four interviewees as they had extensively collaborated with DVN designers and managers. One author moderated Alpha's four employees based on an semi-structured guide that asked the focus group participants to review their DVN consulting projects, workshops, and trainings. Specifically, the participants revisited and discussed anecdotal evidence for lacking DVN design guidance they experienced in their work with practitioners. The focus group participants concluded that DVN designers and managers at DVN orchestrators experience the proposed void of actionable guidance for building DVN design in dealing with value co-creation and emerging networked business environments. The justified problem statement, therefore, is that practitioners 
Table 4 Contexts for Deriving, Illustrating, and Evaluating the Design Principles

\begin{tabular}{|c|c|c|c|c|}
\hline Org. & Context & Purpose & Context Description & Section \\
\hline \multirow[t]{2}{*}{ Alpha } & $\begin{array}{l}\text { Alpha's } \\
\text { R\&D } \\
\text { Project }\end{array}$ & $\begin{array}{l}\text { Deriving } \\
\text { design } \\
\text { princi- } \\
\text { ples }\end{array}$ & $\begin{array}{l}\text { Alpha's co-innovation } \\
\text { project with } 20 \text { se- } \\
\text { nior executives of } \\
\text { European } \\
\text { multi-national enter- } \\
\text { prises that seek to } \\
\text { build DVNs within } \\
\text { this project }\end{array}$ & $\begin{array}{l}\text { Problem } \\
\text { Identificat- } \\
\text { ion and } \\
\text { Eval1 }\end{array}$ \\
\hline & $\begin{array}{c}\text { Alpha's } \\
\text { DVN }\end{array}$ & $\begin{array}{l}\text { Illustrating } \\
\text { design } \\
\text { princi- } \\
\text { ples }\end{array}$ & $\begin{array}{l}\text { Alpha's thriving DVN } \\
\text { for enterprise } \\
\text { software with } \\
\text { 13,000 third parties } \\
\text { that complement } \\
\text { Alpha's core } \\
\text { software package } \\
\text { with software } \\
\text { extensions (e.g., } \\
\text { add-ins, modules, } \\
\text { applications), data, } \\
\text { and consulting } \\
\text { services }\end{array}$ & Appendix 1 \\
\hline Beta & $\begin{array}{l}\text { Beta's } \\
\text { DVN } \\
\text { Design } \\
\text { Works- } \\
\text { hop }\end{array}$ & $\begin{array}{l}\text { Evaluating } \\
\text { design } \\
\text { princi- } \\
\text { ples }\end{array}$ & $\begin{array}{l}\text { Beta, a large steel } \\
\text { producer, build two } \\
\text { principles-informed } \\
\text { DVN designs that } \\
\text { aim to increase } \\
\text { Beta's value } \\
\text { co-creation with its } \\
\text { suppliers, third } \\
\text { parties, and cus- } \\
\text { tomers }\end{array}$ & Appendix 2 \\
\hline
\end{tabular}

lack actionable guidance to efficiently and effectively build DVN designs (EVAL1).

\section{Design and Eval2}

After identifying and evaluating the problem statement in Problem Identification and EVAL1, we anticipated S-D logic as a suitable kernel theory in formulating DVN design requirements. Specifically, we opted for S-D logic's nine derivative propositions (Lusch et al., 2007) as a suitable kernel theory to derive DVN design requirements. Building on S-D logic's foundational premises (FPs), Lusch et al. (2007) derived these propositions as practical implications of the FPs to translate SD logic's descriptive knowledge to more prescriptive knowledge for practitioners (see Table 1). We drew on these nine derivative propositions in formulating DVN design requirements as these propositions' overall theme is to more effectively innovate and compete through multi-actor value cocreation mediated by digital technology. These propositions start from the premise that to "survive and prosper in a networked economy, the organization must learn how to be a vital and sustaining part of the [i.e., organization's] value network" (Lusch et al., 2010, p. 21) (DESIGN).

We evaluated this design decision (i.e., selecting S-D logic's nine derivative propositions as kernel theory for DVN design requirements) in a 90-min long demonstration with seven members of Alpha's R\&D project: two Alpha DVN experts and five executives of major European multinational enterprises. This demonstration was to validate whether the propositions-informed design requirements effectively capture the logic of value co-creation and business environments for building DVN designs. First, one author provided the participants with a primer on S-D logic's nine derivative proposition. Subsequently, this author consecutively discussed the nine derivative propositions with the seven participants to translate them to the context of building DVN designs. Simultaneously, a second author reformulated each DVN design requirement accordingly. EVAL2 revealed that SD logic's nine derivative proposition are a timely, relevant, and useful approach to derive DVN design requirements. We also learnt that, against the backdrop of our far-reaching problem class and design goal, we opt for a high generality level in formulating the design requirements and the design principles to ensure their general applicability for diverse types of DVNs (EVAL2).

\section{Construction and Eval3}

Building on the DVN design requirements, two DVN experts of Alpha's R\&D project and two co-authors of this study prototypically formulated the design principles in a kick-off expert workshop. The workshop participants drew on the DVN design requirements (see Table 8) derived from S-D logic's nine derivative propositions (Lusch et al., 2007) (see Table 1) in formulating prototypical actionable statements as a first attempt to derive the targeted design principles from DVN design knowledge cumulated in Alpha's R\&D project (CONSTRUCTION).

EVAL3 aims to validate the extent to which the drafted actionable statements meet the design requirements. Specifically, we choose an evaluation via expert interviews. Two authors took notes during five semi-structured expert interviews with the same five executives of major European multi-national enterprises that are part of Alphas's R\&D project (see EVAL2). The five interviews were conducted within the frame of two one-day co-innovation workshops of Alpha's R\&D project. Beyond their accessibility and supportiveness, we chose these five interviewees as (1) they confirmed to be unfamiliar with our kernel theory S-D logic; (2) they aimed to initiate new or improve existing DVN designs within Alpha's R\&D project; (3) they already knew the DVN design requirements which was helpful in validating the extent to which the prototypical actionable statements meet these design requirements (see DESIGN and EVAL2). 
Subsequently, all authors analysed and discussed taken notes in a 90-miunte long workshop to reflect learnings in the prototypical design principles. This workshop revealed that the initial design principles' prototypical formulation was immature. The drafted principles (1) were too abstract for and non-applicable by DVN managers and designers due to overemphasizing theoretical and abstract S-D logic language, (2) insufficiently accounted for DVNs' practical requirements, and (3) unstructured in their presentation. We addressed shortcoming (1) (abstractness) by specifically adapting the language to the practitioners' needs. For instance, while the prototypical formulations use the terminology $D V N$ actors, the final formulations use the terminology $D V N$ participant. We addressed shortcoming (2) (void of practical requirements) by complementing the nine DVN design requirements' formulations with practical requirements gathered during the CONSTRUCTION and EVAL3 activities. We addressed shortcoming (3) (unstructured presentation) by following a quadripartite structure for the systematic presentation of design principles in terms of actionable statement, example, rationale, and implications (Aier et al., 2011; Haki \& Legner, 2013; Richardson et al., 1990). Moreover, to overcome a lack of convention regarding the formulation of a design principle's actionable statement, we followed Kruse et al.'s (2015, p. 4045) structure ${ }^{1}$ for design principle formulation (EVAL3).

\section{Use and Eval4}

EVAL4 aims at validating the revised and fully formulated design principles in a naturalistic setting. To this end, we chose a context different from Alpha and turned to Beta, a German multinational conglomerate with focus on industrial engineering and steel production. Beta has limited experiences with building DVN designs and therefore requires actionable guidance in doing so. Specifically, one of the authors organized and subsequently seconded a Beta DVN design workshop in March 2017 to observe Beta employees in building DVN designs. This workshop ideated two DVN ideas to increase their maturity levels in terms of feasibility, viability, and sustainability. It resulted in two documented DVN designs ready for Beta management's investment decision (see Appendices 7 and 8). Therefore, the purpose of Beta's DVN design workshop at hand was to evaluate the proposed design principles' usefulness (in offering actionable guidance in building DVN designs) and effectiveness (in contributing to Beta's competitive advantage).

A total of nine attending Beta employees covered the following positions: business development platforms, product

\footnotetext{
1 "Provide the system with [material property-in terms of form and function] in order for users to [activity of user/group of users - in terms of action], given that [boundary conditions - user group's characteristics or implementation settings]" (Kruse et al., 2015, p. 4045).
}

management, solution management, chief product owner, ecosystem and channels $(\times 2)$; business development, sales head of Europe, custom development. We deem the outlined composition of participants suitable as they combine knowledge of digital technology (e.g., digital platforms), value co-creation (e.g., custom product development), and networked business environments (e.g., ecosystems and channels). We invited a professional moderator which allowed the attending co-author to observe the two groups in using the DVN design principles. Appendix 2 outlines the workshops' detailed process (USE).

During the workshop, the observing co-author recorded the interactions in the form of pictures and personal notes. Specifically, the co-author took notes on when and how the participants relied on the proposed design principles, and whether the current version of their DVN design proffered the action described by the design principle. This account gathered during Beta's DVN design workshop presents preliminary evidence that the design principles are useful and effective. However, more data is needed to be able to conclude the design principles' usefulness and effectiveness (EVAL4). Next, we report on the design of our EVAL4.

Usefulness We aim at validating the design principles' actionability to evaluate their usefulness. Actionability here captures the extent to which the design principles offer actionable guidance to the actual user of the design principles - the builder of a DVN design. A designer follows the proposed design principles' actionable statements to build a DVN design. Therefore, we evaluate in how far a design principle is actionable by the DVN designer. Specifically, we measure whether-and how efficient - the design principles can be instantiated into a concrete DVN design, and whether this DVN design indeed proffers the action described by the design principle (Kruse et al., 2015). Guided by the design principles, the DVN design workshop participants built two DVNs designs - hall building platform (see Appendix 7) and steel slug platform (see Appendix 8). We present these two DVN designs in detail in the section Design Principles Evaluation: Beta's DVN Design Workshop to provide evidence for the DVN design principles' actionability.

Effectiveness We ultimately aim at validating how effectively the design principles improve an organization's competitive advantage. To wit, our kernel theory S-D logic and its set of derivative propositions inform practitioners about improving competitive advantage through a service logic (Lusch et al., 2007). The targeted design principles aim to increase competitive advantage through the realization of principles-informed DVN designs. As measuring competitive advantage that is attained through the application of the DVN design principles requires a longitudinal study that is beyond the scope of this research. Instead, we collect evidence for whether the principles-informed DVN 
designs from Beta's workshop facilitate Beta's learning and change. Specifically, we conducted a one-hour semistructured telephone interview with a Beta employee who had attended Beta's DVN design workshop. The workshop resulted in two documented DVN designs. They served as basis for an investment decision. The principles-informed DVN designs were considered a high-quality basis for the discussion with Beta management (learning). In this respect, the interviewee reported that the DVN designs provided the following advantages: (1) learning about a more complete and nuanced compilation of relevant DVN design features, (2) learning about a more precise description of relationships between these DVN design features, and (3) learning about the identification of a high number of crucial challenges in the DVN designs that served as pivotal points of discussion with Beta management.

\section{Results}

Guided by Sonnenberg and vom Brocke's (2012, p. 392) cyclic DSR process (see section Research Method), we identify nine DVN design requirements and four DVN design principles. DVN design requirements represent the problem space of DVN design in that they capture generic requirements that any instance of DVNs should meet to function effectively (Baskerville \& Pries-Heje, 2010; Walls et al., 1992). DVN design principles embody prescriptive knowledge that bridges the problem space (DVN design requirements) and solution space (DVN design features) of DVN design (Gregor \& Hevner, 2013; Kruse et al., 2015). Design principles, thus, serve as a means to convey design knowledge that contributes beyond context-bound DVN instantiations (Kruse et al., 2015). Design principles also constitute general solution components technologies that can be instantiated into several exemplars of DVNs (Iivari, 2015). Table 8 synthesizes the resultant requirements and principles for DVN design — all of which we present in this section.

\section{Design requirements for digital value co-creation networks}

Relying on our research method's Design and Eval2 phase, we identify DVN design requirements that represent the problem space of DVN design. Our understanding of design requirements is closely associated with the meta-requirements (Walls et al., 1992) and general requirements (Baskerville \& PriesHeje, 2010) concepts. Design requirements in this sense comprise generic requirements that any DVN instantiated from this design should meet. This section outlines the identified DVN design requirements (DRs) in association to S-D logic's derivative propositions (DPs) (Lusch et al., 2007, p. 8). We use the notion $D P n$ to refer to the $\mathrm{n}^{\text {th }}$ derivative proposition of S-D logic and present the respective proposition in italics.

DR1. DVNs should enhance their participating organizations' competitive advantages by applying operant resources more effectively than non-participating organizations S-D logic specifies that competitive advantage is a function of how the firm applies its operant resources to meet the needs of the customer relative to how another firm applies its operant resources (DP1). Organizations that have access to suitable operant resources gain enhanced opportunities for differentiation from their competitors. DVNs should regard resources as an essential component and the basis for value generation. Hence, we propose DVNs to enable an effective mobilization of operant resources to increase the competitive advantage of their participating organizations in differentiation to nonparticipating organizations.

DR2. DVNs should integrate operant resources between participating organizations in designing digital service S-D logic describes collaborative competence as a primary determinant of a firm's acquiring the knowledge for competitive advantage (DP2). Such collaborative competence refers to a central component of an organization's network (i.e., suppliers, partners, customers, and competitors) (Wirtz et al., 2016) and the specific actor roles in the DVN. The competence to set up this network effectively (e.g., attracting augmenting third party in satisfactory quality and quantity) is crucial for DVN survival as this competence ensures to ultimately integrate resources between these actors.

DR3. DVNs should embrace information technology as an operant resource to initiate value co-creation processes S-D logic suggests that operant resources are "the fundamental source of competitive advantage" (Vargo \& Lusch, 2008, p. 7). Specifically, S-D logic points out that the continued ascendance of information technology with associated decrease in communication and computation costs, provides firms opportunities for increased competitive advantage through innovative collaboration (DP3). Recent IS research conceptualizes novel IT (e.g., data mining, prescriptive analytics) as operant resource in that it "seek[s] out and pursue[s] unique resource integration opportunities on its own, and in the process, engage[s] with (or act[s] upon) other actors" (Lusch and Nambisan 2015, p. 167). DVN should, thus, embrace novel IT as operant resource to play an active role in triggering or initiating value co-creation and in affecting DVN participants and their choices (Vargo and Lusch 2004; Akaka and Vargo 2014). From an S-D logic perspective, embracing IT as operant resource combined with the plethora of data available in the digital economy (Ross et al., 2016) affords competitive advantage for DVN participants. Moreover, data can be completely decoupled and shared independent of the original 
physical good with little or no costs and time-delay of physical transport. Against this backdrop, DVNs should focus on the specific opportunities of digitalization (Loebbecke \& Picot, 2015) through comprising operan $t$ IT in the design, commercialization, and monetarization of digital service (Barrett et al., 2015). This can even lead to the situation that a traditional business model (e.g., Beta's business model of selling offthe-shelf steel) is completely overhauled by technologyenabled value propositions that grasp the opportunities of IT (Loebbecke \& Picot, 2015). Service science has already emphasized the fundamentally novel role of IT in the digital economy (Lusch \& Nambisan, 2015) and the role of novel and complex service-based business models (Maglio \& Spohrer, 2013) that fully utilize the opportunities of IT.

DR4. DVNs should engage end customers and third-party actors in value co-creation activities In G-D logic, value creation is a function of linear processes starting from simple basic components and ending in packaged multicomponent goods. Conversely, in S-D logic, value creation is a function of multi-directional processes in multiactor constellations entailing multiple, varied, and interdependent actors. DP4 states that firms gain competitive advantage by engaging customers and value network partners in co-creation and co-production activities. Also, effective mobilization of resources within the DVN is here viewed as a unique source of competitive advantage. Mobilization, in turn, refers to facilitating the best combination of resources for a particular situationthat is, for a customer at a given time in a given place - to create the optimum value/cost result (Lusch \& Nambisan, 2015). DVN design, thus, calls for various available resources of different suppliers and partners to be mobilized from and to pivotal actors. To illustrate, distributed slack resources (e.g., an unused car ready for sharing) can be better utilized than in traditional business approaches, partially by the effective access to third-party, noncommercial car providers.

DR5. DVNs should rely on third-party actors to understand how end customers uniquely integrate resources Value cocreation is related to more intensive interactions between actors (value-in-use) and goes far beyond the traditional logic of production and delivery of goods (value-in-exchange) (Baines, 2015). S-D logic's DP5 states that understanding how a beneficiary uniquely integrates her resources and experiences service (both private and public) is a source of competitive advantage through innovation. In a similar vein, business model research underscores the value of understanding end customers by means of value propositions (Wirtz et al., 2016). In addition to value propositions, customer journeys (Stickdorn \& Schwarzenberger, 2016) effectively model the process of how customers use a service; they help improve digital service delivery by better addressing the customers' context. In DVN, third parties - with their industry competence, end-user-specific knowledge, close relationships with end user organizations, and reach to end user organizations in any geographical location-are well-positioned to conceive how end customer organizations uniquely integrate resources.

DR6. DVNs should provide service co-design opportunities to end customers and third-party actors at once S-D logic's DP6 states that providing service co-production opportunities and resources consistent with the customer's desired level of involvement leads to improved competitive advantage through enhanced customer experience. This points to the fact that service beneficiary involvement is not only a burden, but this involvement can increase the value of a digital service. In this way, DP6 requires DVNs to motivate and facilitate end customers and third-party actors to participate in service codesign activities. Effective DVN design, thus, calls for such service $c o$-design via customer journeys as part of the customer relationship.

DR7. DVNs should adopt collaboratively developed, riskbased pricing and cost mechanisms DP7 informs us that firms can compete more effectively through the adoption of collaboratively developed, risk-based pricing value propositions. DR7 refers to an organization's revenue model (i.e., revenue streams and pricing mechanisms) and financial model (i.e., financing model, capital model, and cost structure model) (Wirtz et al., 2016). Effective DVN design, thus, should ensure that economic risks, costs, and revenues are fairly distributed among the multitude of its participating actors. This design requirement ensures that a DVN runs and evolves in a stable manner.

DR8. DVNs should be orchestrated by a prime resource integrator $D P 8$ tells us that the value network member that is the prime integrator is in a stronger competitive position. While this DP8 only underscores that initiating and orchestrating a service ecosystem yields in competitive advantage, participants in our design requirements' demonstration (Design and Eval2) did emphasize that effective resource integration in DVNs demands a prime resource integrator. This integrator orchestrates third parties that are distributed temporally, organizationally, and spatially. DR8 emphasizes the leading role of the ecosystem's prime resource integrator (or orchestrator) as the central actor who determines exchange protocols, third-party quality standards, and the roles of DVN participants in the orchestrator's business model. Thus, DVN orchestrators must pay attention to the distribution of roles within the DVN to improve the network's operation assuring their own primary role. 
DR9. DVNs should treat their participating organizations' employees as operant resources in designing digital service With respect to an organization's internal knowledge, S-D logic specifies that firms that treat their employees as operant resources will be able to develop more innovative knowledge and skills and, thus, gain competitive advantage (DP9). IT facilitates the effective mobilization of such internal key resources within an organization. In this way, mobilizing previously neglected, latent internal resources now becomes essential for designing innovative digital service in DVNs. DVNs should treat their participating organizations' employees as operant resources.

\section{Design principles for digital value co-creation networks}

Relying on our research method's Construction and Eval3 and Use and Eval4 phases, we identify four generic DVN design principles. Our understanding of design principles is closely related to the meta-design (Walls et al., 1992) and general components (Baskerville \& Pries-Heje, 2010) concepts. Design principles, therefore, convey design knowledge that contributes beyond context-bound DVN instantiations (Kruse et al., 2015). Design principles constitute a general solution that can be instantiated into several DVN exemplars (Iivari, 2015). Table 4 summarizes the four DVN design principles and their relations to DVN design requirements. The four design principles do not merely guide DVN design in isolation. Conversely, they are interrelated in that they mutually support and complement one another. Consequently, the recognition of interrelations between design principles plays an essential role in DVN design as outlined in Table 5 (see column Design Principle's Interrelatedness). This section describes the DVN design principles that can be applied to realize the shift to S-D logic through IS. Our intent here is not only to capture the general design guidance for DVNs, but also to illustrate their implications for organizational practice.

Each principle is discussed in detail following the design principle's actionable statement, example, rationale, and implications (Aier et al., 2011; Richardson et al., 1990). To overcome a lack of convention as to how a design principle's actionable statement should be formulated and what exactly a design principle is, we follow Kruse et al.'s (2015, p. 4045) structure for design principle formulation: "Provide the system with [material property-in terms of form and function] in order for users to [activity of user/group of users-in terms of action], given that [boundary conditions-user group's characteristics or implementation settings]."

\section{The principle of ecosystem-oriented design}

Actionable statement Provide the DVN with control mechanisms and specific third-party roles for DVN participants (1) to align the operations of third-party resource integration and (2) to ensure fair sharing of economic risks, costs, and revenues among all DVN participants, given that DVNs are structurally and dynamically complex multi-actor settings.

Example A prime example of ecosystem-oriented design are the multi-sided markets that are attractive to service beneficiaries because of the multitude of service offerors, and vice versa (Tan et al., 2015). For instance, multi-sided markets play a vital role in mobile payment ecosystems (Kazan et al., 2018). Such mobile payment ecosystems are contingent on a critical mass of actors within each actor role (e.g., banks, payment platforms, service offerors, service beneficiaries). Payment ecosystems benefit from cross-sided network effects: a beneficiaries' adoption of mobile payment increases value for offerors and financial institutions, and vice versa $(\mathrm{Du}$, 2017). A prime network orchestrator often plays a vital role in such networks (Loukis et al., 2016). This orchestrator provides services to the interacting parties and benefits from controlling network access. Electronic marketplaces demonstrate this principle. The orchestrator can stimulate the network by providing additional services that cater for targeted information or enable transactions. This bestows an extraordinary market position on the orchestrator.

Rationale The structure of business environments changes from a directed sequence - the supply chain - to a dynamically reconfiguring network of mostly loosely coupled actors who interact in various directions. The DVN facilitates the reconfiguring connections and enables actors to interact with each other. DVNs should, thus, account (1) for orchestration of specific actor roles in a service ecosystem; (2) for positioning of an organization's role as focal orchestrator in a service ecosystem; as well as (3) for sharing of economic risks, costs, and revenues among a multitude of various actor roles in a service ecosystem.

Overall, S-D logic emphasizes the actor-to-actor network as the location of economic exchange (Lusch \& Nambisan, 2015). Based on collaboration and competition, dynamic and co-evolving communities allow the various actors to work together and to create and capture new value. It is especially the motivation of actors in service ecosystems that is crucial for effective DVN operation. It is, hence, the orchestrators' task to stimulate their motivation and support direction to joint DVN operations.

In contrast to G-D logic, where value is mainly created in the single organization, actor-to-actor networks follow an increasingly diverse and complex S-D logic of value 
Table 5 Design Principles for Digital Value Co-Creation Networks

Design Principles (Ps) in Design Principle's Actionable Statement Association to Design

Requirements (DRs)

P1. Principle of

Ecosystem-Oriented

Design

$(D R 2, D R 7, D R 8)$

P2. Principle of

Technology-Oriented

Design

$(D R 3, D R 4)$

P3. Principle of

Mobilization-Oriented

Design

(DR1, DR2, DR4, DR9)

P4. Principle of

Interaction-Oriented

Design

(DR4, DR5, DR6, DR7)
- Provide the DVN with control mechanisms and specific third-party roles for DVN participants

-(1) to align the operations of third-party resource integration and (2) to ensure fair sharing of economic risks, costs, and revenues among all DVN participants,

- given that DVNs are structurally and dynamically complex • Extensive interaction is required to ensure an ecosystem's multi-actor settings.

- Provide the DVN with operant information technology for - An ecosystem includes DVN participants that provide DVN participants

- (1) to exploit data inter-organizationally, (2) to separate informational assets from physical goods, and (3) to facilitate the commercialization of both through digital channels,

- given that DVN participants are distributed temporally, organizationally, and spatially.

- Provide the DVN with transparency mechanisms for DVN participants

- to identify and mobilize own (internal) and third-party (external) resources in innovating and designing digital service,

- given that DVNs expeditiously mobilize distributed resources for any given end customer context.

- Provide the DVN with interaction opportunities and a protocol of exchange for DVN participants

- to engage in mutual value co-creation activities,

- given that DVNs hold heterogeneous and complementary resources at DVN orchestrators, third parties, and end customers.
Design Principle's Interrelatedness

- Technology helps build and maintain connections between DVN participants $(P 2)$.

Mutual resource mobilization provides a driver for establishing, stabilizing, and diversifying an ecosystem (P3).

survival $(P 4)$ new technology or that adopt existing technology employed by the DVN owner to mediate its DVN $(P l)$.

- Technologies are contingent on mobilizing meaningful digital data without which effective and efficient digital service is hardly feasible $(P 3)$.

- Interaction amongst DVN participants yields in evolving third-party or end-customer requirements contingent upon new technologies (P4).

- An ecosystem makes a broad variety of resources available for mobilization in the first place $(P 1)$.

- Technology mediates the mobilization and integration of distributed resources for any given end customer context (P2).

- New resources result from the DVN participants' ecosystemic interaction $(P 4)$, such as digital data as the central resource of a DVN.

- Interaction is contingent on an ecosystem of third parties and end customers all of which are available for mutual interaction $(P l)$.

- Technology mediates efficient and effective interaction amongst DVN participants $(P 2)$.

- In the process of mobilizing resources, DVN participants interact to identify and exploit relevant resources $(P 3)$. (co-)creation. Whereas G-D logic mainly focuses on resource supply, the collaborative elements of networks are mainly ignored. According to Zott and Amit (2008), business models play a central role for understanding the orchestrating of service ecosystems. Particularly, Leminen et al. (2012) point to the role of business models in explaining the opportunities of digital service ecosystems. However, most business model representations concentrate on the focal organization and regard service ecosystems only implicitly via the interfaces to immediate partners and customers. DVNs that realize multisided business models, however, require proper network representations.

Implications (1) The design of DVNs needs to extend the focus from processes, activities, resources, and practices within an organization to a coordination and governance of service ecosystems; (2) the roles in DVNs must be defined properly to facilitate value co-creation; and (3) the motivation of actors assuming these roles (e.g., service offeror, service beneficiary, ideator, designer, and intermediary) need to be considered and properly addressed. Value co-creation aims at providing benefits to all actors whose collaboration in the DVN is required.

\section{The principle of technology-oriented design}

Actionable statement Provide the DVN with operan $t$ information technology for DVN participants (1) to exploit data interorganizationally, (2) to separate informational assets from physical goods, and (3) to facilitate the commercialization of both through digital channels, given that DVN participants are distributed temporally, organizationally, and spatially.

Example The large number of actors and the plethora of data in DVNs can only be handled by means of IT, such as traditional and advanced analytics techniques (Ngai et al., 2017). Two prime examples in this respect are (1) the Internet of Things for capturing data and (2) machine learning for aggregating 
data (Loebbecke \& Picot, 2015). We find a growing number of smart devices, which are digitally connected to other artifacts and realize sophisticated business scenarios in manufacturing, mobility, or healthcare (Beverungen et al., 2017). Technology also yields positive effects in offering a variety of online channels for mobile or electronic commerce (Chen et al., 2014; Jeansson et al., 2017). To exemplify further, electronic marketplaces rely on the efficacy of search and navigation services that support customers in finding the right product among the multitude of offerings. In these marketplaces, technology is needed to guide customers efficiently through procedures such as financial transactions or the input for delivery services with their variety of options.

Rationale The role of IT is changing from focusing on the efficiency of an organization's internal processes to enabling effective coordination and exchange of data between organizations (Karhu et al., 2018; Lusch \& Nambisan, 2015). This comprises diverse options of capturing, aggregating, and distributing data between organizations via IT. DVN participants rely on IT to embrace these diverse options through ITmediated interactions. Effective DVN design, thus, is contingent on IT, organized in a digital infrastructure, for decoupling informational assets from products and facilitating their commercialization; as well as for driving value creation through digital channels and digitally enhanced customer relationships.

Implication (1) Organizations require a fresh view on IT and digital resources to design and innovate digital service using new operant resources (e.g., data, information, knowledge, experience, skills); (2) Organizations need to concentrate on the decoupling of informational assets from physical goods, the de-linking of data ownership and value creation and, finally, the development of collaborative competence out of systematic use of IT; (3) Organizations need to consider IT to ensure the basic operation of DVNs, providing all DVN participants with the functionality to accomplish their particular role.

\section{The principle of mobilization-oriented design}

Actionable statement Provide the DVN with transparency mechanisms for DVN participants to identify and mobilize own (internal) and third-party (external) resources in innovating and designing digital service, given that DVNs expeditiously mobilize distributed resources for any given end customer context.

Example Organizations that allow for transparency of all available operan $t$ resources in their digital ecosystem increase the chance of interaction between actors. Airbnb and Uber, which make use of the partners' unused apartments, vehicles, and workforce, are an example for this trend. The mobilization of knowledge which apartments, vehicles, and workforce are available at a given time, and the skills on how to combine this knowledge yield effective access to resources to beneficiaries. In future, we can expect the mobilization of other operant resources that we cannot even imagine today. If actors realize such interactions, DVNs (re)form. Since such DVNs can be quite large, even rarely requested resources might be mobilized and find interested parties, leading to benefits for offerors as well as beneficiaries. For example, popular electronic marketplaces, with their efficient search services and reduced transaction costs, make long-tail products available, which otherwise would not find an appropriate market (Brynjolfsson et al., 2011). Similarly, social networking platforms such as LinkedIn or XING simplify the mobilization of job seekers and job offerors (Buettner, 2017).

Rationale Previously, static acquisition and processing of properly selected operand resources is replaced by the dynamic invitation and brokerage of operant resources that DVN participants make available to each other. Consequently, more resources are available for mobilization. The DVN must enable the identification, activation, distribution, and utilization of DVN participants' resources. Effective DVN design, thus, is contingent on transparent access to and mobilization of operant resources for uncovering and utilizing DVN participants' internal knowledge ultimately. This new view on thirdparty actors and end customers' (external) as well as employees' (internal) operant resources suggest the creation of new digital services by revising DVNs' value creation, proposition, delivery, and capture. Moreover, digital technology leads to a dramatic reduction of transaction and coordination costs, another source for new business opportunities through mobilizing operant resources.

Implication In designing DVNs, (1) organizations must aim at the activation of unused internal and external operant resources changing existing or creating completely new digital service(s); (2) technology facilitates activation through increasing resource density; and (3) organizations must consider inter-organizational coordination mechanisms that help with mobilizing and activating resources that are distributed in the DVN. Such inter-organizational activation of resources is likely to yield an enhanced competitive edge for DVN participants.

\section{The principle of interaction-oriented design}

Actionable statement Provide the DVN with interaction opportunities and a protocol of exchange for DVN participants to engage in mutual value co-creation activities, given that DVNs hold heterogeneous and complementary resources at DVN orchestrators, third parties, and end customers. 
Example The acceleration of innovation processes (Lyytinen et al., 2016) leads to an entwinement of service design, innovation, and consumption (Perks et al., 2012). For instance, beneficiaries give detailed feedback or even enable new opportunities, such as McDonald's MyBurger initiative or Lego's Ambassador Program. Likewise, interaction in social media plays an increasingly key role in gaining information about customer behavior and yields valuable insights on customers (Baumol et al., 2016; Wieneke \& Lehrer, 2016). Analyzing these interactions has become an important tool for customer orientation (Alt, 2016). Feedback in social media is not only a suitable channel to express satisfaction or discontent with the service and the brand (Kabadayi \& Price, 2014), it has also become a major means to increase service transparency (Hollebeek, 2013). The example of car sharing shows that the benefit does not only lie in providing a car, but that it also comes along with other values, namely that the most suitable car is available at the right time and at the right place, while burdens such as looking for parking possibilities are reduced, for example, by the provision of specific local parking slots. Finally, interaction itself generates further data that can then be utilized by DVN participants.

Rationale By closer and more timely interaction, service provisioning can be adapted more precisely to beneficiaries' needs. Consequently, DVNs must aim at establishing continuous reciprocal exchange instead of single transactions. Effective DVN design should account (1) for beneficiary and thirdparty involvement, enhancing value-in-use, and sustaining beneficiary and third-party engagement; (2) for reflecting on value co-creation through customer journeys as dynamic interaction; as well as (3) for recalibrating service bundles to optimize customer experience. Although economic actors have always interacted to integrated resources, we observed most of these interactions inside the organization. DVNs have led to extensive interaction beyond organizational boundaries (Barrett et al., 2015; Lusch \& Nambisan, 2015; Srivastava \& Shainesh, 2015). Such boundary-spanning interaction comes along with the advantage of value $\mathrm{Co}$-creation to address beneficiary needs more effectively. Through close and timely the interaction, DVNs integrate a service beneficiary, the DVN orchestrator, and third parties to improve the beneficiary's value-in-use. As such, beneficiaries profit significantly from today's service economy as interaction within DVNs allows for more contextualized digital services.

Implication (1) DVNs that are based on the analysis of a particular beneficiary's objectives and the available resources can facilitate an interaction that significantly enhances the beneficiary's value-in-use; (2) the involvement of customers beyond accepting value propositions through new channels of interaction must aim at the optimization of the beneficiary experience by the most desirable combination of resources; and (3) visual inquiry tools such as customer journeys help to move away from static value propositions and design dynamic interactions, which blueprint how service beneficiaries may engage in value co-creation and which benefit they can receive.

\section{Illustration and evaluation}

This section relates to the organizations Alpha and Beta to illustrate and evaluate the proposed design principles, respectively. While Alpha's DVN (see Appendix 1) serves as illustration of the design principles (through highlighting its DVN's expository design features), Beta's DVN design workshop (see Appendix 2) serves as naturalistic evaluation (by using the derived design principles in Beta's DVN design workshop) as Beta lacks experience and actionable guidance in building DVN designs.

\section{Design principles illustration: Alpha's DVN}

Alpha's DVN instance has been useful in operating a thriving business-to-business digital platform for enterprise software that has survived over a prolonged period since 2012 (see Appendix 1). As digital, multi-actor value co-creation processes among the constituent actors of Alpha's platform are (1) a pivotal antecedent for the survival of Alpha's platform and (2) receive increasing attention in digital platform research (Lusch \& Nambisan, 2015), we investigate four expository design features of Alpha's DVN that are pivotal for Alpha's platform success. In the specific context of digital platforms, the proposed design principles - derived within Alpha's R\&D project and based on S-D logic's nine derivative proposition (Lusch et al., 2007) - guide those who are interested in designing digital platforms for digital, multi-actor value co-creation among the platform's constituent actors.

In turn, the proposed DVN design features belong to the solution space of DVN design in that they denote specific technical ways to implement a design principle in an actual DVN instance (Meth et al., 2015). While design principles abstract from technical specificities, design features explain why a technical specificity leads to a specific goal. Therefore, while the presented design requirements and principles are exhaustive in their derivation from the employed kernel theory, the design features are solely discussed as exemplary technical features that are specific to Alpha's DVN. In the context of this paper, these illustrative DVN design features denote specific technical ways to implement the general DVN design principles in Alpha's DVN instance. Table 6 summarizes the four DVN design features and their association to the DVN design principles. In turn, Table 8 
Table 6 Design Features of Alpha's DVN

\begin{tabular}{|c|c|}
\hline $\begin{array}{l}\text { Design Features (DFs) in Association to } \\
\text { Design Principles (Ps) }\end{array}$ & Definition \\
\hline $\begin{array}{l}\text { DF1. Extensible Codebase for } \\
\text { Enterprise Software }(P 1, P 2)\end{array}$ & $\begin{array}{l}\text { Semi-open enterprise software serving as a building block upon } \\
\text { which third-party derivatives can be added }\end{array}$ \\
\hline $\begin{array}{l}\text { DF2. Enterprise Software Derivatives } \\
\quad(P 1, P 4)\end{array}$ & $\begin{array}{l}\text { Peripheral third-party software, hardware, or service augmenting a } \\
\text { core extensible codebase }\end{array}$ \\
\hline $\begin{array}{l}\text { DF3. Integration and Certification } \\
\text { Center }(P 2, P 3)\end{array}$ & $\begin{array}{l}\text { Quality management program certifying peripheral third-party de- } \\
\text { rivatives that integrate with a core extensible codebase }\end{array}$ \\
\hline $\begin{array}{l}\text { DF4. API Management Software }(P 2 \text {, } \\
\quad P 3)\end{array}$ & $\begin{array}{l}\text { Standard enterprise software to organize defined exchange protocols } \\
\text { for inter-systems communication }\end{array}$ \\
\hline
\end{tabular}

summarizes the tripartite organizing structure of interrelated requirements, principles, and features for DVN design.

DF1. Extensible codebase for Enterprise software Alpha provides its DVN with an extensible codebase for enterprise software that is accessible to third parties via an open platform as a service (PaaS). Extensible codebase for enterprise software here refers to semi-open enterprise software serving as a building block upon which third-party derivatives can be added (Parker et al., 2017). By means of its extensible codebase, Alpha's DVN implements the principle of ecosystem-oriented design (P1): Alpha's extensible codebase integrates different software (e.g., add-ins, modules, apps), hardware, and service extensions, and thereby tracks all value co-creation activities among Alpha's DVN participants. Examples are on- and offboarding activities for DVN participants, cash flows, API requests, registrations, or third-party log-ins. The extensible codebase serves to ensure fair sharing of economic risks, costs, and revenues among all DVN participants. DF1 also implements technology-oriented design (P2): Alpha's extensible codebase provides circa 13,000 third parties and circa 130 end user organizations with the facilitating information technology to synergistically integrate their distributed resources. Such extensible codebases extend value creation in firms beyond their organizational boundaries (Parker et al. 2017; de Reuver et al. 2018). Appendix 3 illustrates Alpha's extensible codebase for enterprise software, the first expository design feature of Alpha's DVN.

DF2. Enterprise software derivatives Alpha provides its DVN with a sophisticated program for enterprise software derivatives, which we here refer to as peripheral third-party software (e.g., add-ins), hardware (e.g., end user devices), or service (e.g., database migration) augmenting a core extensible codebase. These derivatives comprise four dedicated thirdparty engagement modes to augment Alpha's enterprise software: build, run, sell, service. Build derivatives include applications, software extensions (e.g., add-ins, modules, mobile apps), and integrated solutions to complement Alpha's standard software. Run derivatives offer private- or public-cloud- deployed services to end user organizations. Sell derivatives refer to third parties that resell Alpha solutions while managing an entire service's lifecycle at the end user organizations. Service derivatives refer to third parties that advise Alpha's end user organizations in implementing Alpha's enterprise software. These enterprise software derivatives, a design feature of Alpha's DVN, implement the principles of ecosystemoriented design (P1): the four distinct derivatives (i.e., build, run, sell, and service derivatives) allow Alpha to provide its DVN with specific third-party roles for DVN participants (1) to identify the right third party in the DVN and (2) to allocate economic risks, costs, and revenues among all actors, contingent on their respective roles (P1). DF2 also implements the principle of interaction-oriented design (P4): the four distinct types of enterprise software derivatives provide Alpha's DVN with a protocol of exchange for its DVN participants to interact with different derivative types differently. For instance, to exchange with service partners (e.g., Accenture) is different than to exchange with build partners (e.g., Salesforce) (P4). Appendix 4 illustrates the enterprise software derivatives, the second expository design feature of Alpha's DVN.

DF3. Integration and certification center Alpha's integration and certification center (ICC) bridges DF1 (Extensible Codebase for Enterprise Software) and DF2 (Enterprise Software Derivatives). That is, ICC was established in 1996 to provide services around the integration of third parties' enterprise software derivatives with Alpha's extensible codebase. ICC here refers to a quality management program certifying peripheral third-party derivatives that integrate with a core extensible codebase (Eaton et al., 2015). Alpha offers ICC as an open program for any third party in its DVN (e.g., independent software vendor, consultancies) that wishes to have their derivative certified with the latest Alpha technologies. Thereby, Alpha's ICC facilitates seamless integration of Alpha's DVN participants, shortens implementation times, reduces integration costs, and achieves compatibility with the digital infrastructure of end user organizations using Alpha technology. Beyond these technological aspects, Alpha's ICC facilitates a high-quality DVN of third-party derivatives 
that contribute to the comprehensive value proposition and innovation sources for end user organizations. Moreover, Alpha's ICC allows third parties to leverage Alpha branding and momentum, and it helps DVN participants to accelerate the market ramp-up cycle. Once Alpha has signed a contract to certify a third-party derivative's integration, the third party will be assigned a dedicated Alpha consultant. The third party's engineers will prepare the testing under the guidance of this consultant and schedule a certification date. During any certification test, the Alpha consultant monitors and logs the test results, while the third-party engineers drive the actual testing, including the integration set-up and data exchange. Once the testing is concluded successfully, the third party receives a detailed and confidential test report, a certificate, and the appropriate certification logo to promote the successful certification to end user organizations. Any fully certified solution is listed online in Alpha's Certified Solutions Directory. Serving as a business-to-business sales channel for enterprise software derivatives, the directory gives certified third parties exposure to a large set of end customer organizations that visit Alpha's Certified Solutions Directory each day (see Appendix 5). The ICC design feature implements the principle of technology-oriented design (P2): The Certified Solutions Directory serves as technical means for DVN participants to commercialize platform-augmenting derivatives (e.g., software modules, data, or consulting services) through an additional digital channel operated by Alpha. DF3 implements mobilization-oriented design (P3): the Certified Solutions Directory provides DVN participants an easy-tonavigate overview of all readily-available and certified resources in Alpha's DVN. Appendix 5 illustrates this easy-tonavigate overview in Alpha's Certified Solutions Directory. Once an end user organization identifies a required resource (e.g., a niche software module), it can start an interaction with the third party. An ICC design feature is particularly relevant given that DVNs integrate heterogeneous and distributed resources of DVN orchestrators, third parties, and end user organizations. Appendix 5 illustrates Alpha's ICC, the third expository design feature of Alpha's DVN.

DF4. API management software Alpha provides its DVN participants with a dedicated standard software product for application programming interfaces (APIs) management, which we here refer to as standard enterprise software to organize defined exchange protocols for inter-systems communication. Alpha's DVN participants pervasively expose their own internal and draw on external APIs in facilitating their digital multi-actor value co-creation processes. These heterogeneous and distributed sets of internal and external APIs need to be carefully integrated and managed within each DVN participant in (re)forming relations to other DVN participants. In turn, API here refers to a defined exchange protocol to facilitate inter-systems communication (Tiwana, 2015). DF4 addresses DVN participants' API needs such as API provisioning and publishing, API discovery and consumption, as well as API security and access control. DF4 provides a framework using REST, OData, and standard SOAP services to expose Alpha or non-Alpha backend data and processes. By means of its API management software, DF4 implements the principles of technology-oriented design (P2): Alpha's API management software provides pivotal technical capabilities for two seminal audiences in its DVN. While service providers create API proxies and API products to expose backend services, platform extension developers (Alpha build partners) consume these API proxies and products to create platform-augmenting extensions for mobile and desktop devices. Moreover, developers at Alpha's end user organizations also use the API management software to integrate external and expose internal APIs to their customers and third parties. To this end, the API management software's user interface provides browser-based tooling for DVN participants to create, configure, and manage API proxies and products. DF4 also implements mobilization-oriented design (P3): the API management software in Alpha's DVN allows third-party extension developers and end customer organizations to identify and interface with distributed extensions for any given end customer context. Appendix 6 illustrates Alpha's API management software, the fourth and last expository design feature of Alpha's DVN. While this section showcases expository design features of Alpha's DVN to illustrate the proposed DVN design principles, the next section presents the results of Beta's DVN design workshop as a naturalistic evaluation.

\section{Design principles evaluation: Beta's DVN design workshop}

Our analysis of Beta's DVN design workshop (see EVAL4 and Appendix 2) suggests that the design principles helped the workshop participants to instantiate two DVN designs. Due to the nature of the artifact, the EVAL4 remains preliminary. While our preliminary EVAL 4 presents evidence that the design principles are useful and effective to some degree, more data is needed to be able to conclude it. We provide an intermediate contribution in the knowledge accumulation and evolution in DSR which shall be picked up and extended by future research (vom Brocke et al., 2019). Next, we report the preliminary results of our EVAL 4 .

The first principles-informed DVN design, a hall building platform (see Appendix 7), mediates the integration of a hall building project's activities (i.e., hall planning, product decisions, scheduling, ordering, production, delivery of prefabricated and finished materials for assembly). The second principles-informed DVN design, a steel slug platform (see Appendix 8), mediates Beta's inhouse laser cutting of shaped steel slugs ordered by different customers on a single steel coil to reduce steel waste. Second, we investigate whether these 
two DVN designs proffer the actions described by the design principles as outlined in Table 7. Finally, we observed that the design principles' outlined interrelatedness helped participants to design features that relate to at least two design principles. For example, this particularly concerned three design features of Beta's Hall Building Platform: digital platform, online marketplace, and engagement program (see Table 7). At the workshop's outset, the participants already had an idea of these three features. Relating to the four principles, the participants relied on several principles in designing the same feature:

- Digital Platform: (a) how to collect distributed digital data (mobilization) via a digital platform (technology); and (b) how interaction between customers on the portal could be used to leverage data.

- Online Marketplace: (a) how to use the online marketplace to access resources in the ecosystem (mobilization); (b) how to foster interaction on the online marketplace; and (c) how to use the digital platform functionalities (technology) to support interaction on the portal.

- Engagement Program: (a) how to enable value co-creation between Beta, third parties, and customers through the exchange of data (interaction); and (b) how to transparently showcase all third parties and customers on the online marketplace (mobilization) to attract further third parties and customers (ecosystem).

We observed that the four design principles played a crucial role in designing these three features. The design principles did not only serve in pointing to essential features of Beta's hall building platform, but also in pointing to complimentary combinations of the informing design principles. Thus, the design principles have proven actionable for the designers as the principles complementarily inform one another. The workshop revealed that, prima facie, the design principles were not entirely unfamiliar to participants, but the fact that they exposed additional underlying structure gave workshop participants guidance towards complementary combinations that they deemed particularly useful.

\section{Discussion and conclusion}

The central outcome of this design science research is prescriptive knowledge in the form of design requirements and design principles for digital value co-creation networks (DVNs) - defined as complex, socio-technical service ecosystems to configure emergent, networked, and IS-enabled value co-creation processes resulting in digital service. The design requirements are built upon service-dominant (S-D) logic's derivative propositions (Lusch et al., 2007)—representing managerial implications of S-D logic. The design principles, namely ecosystem-, technology-, mobilization-, and interaction-oriented design, represent a general solution for DVN design that addresses the DVN design requirements. Alpha's thriving DVN case illustrated how to map the generic DVN design principles to specific DVN design features. Table 8 organizes the interrelated requirements, principles, and features for DVN design. Our core contribution is a set of four DVN design principles to guide organizations in building DVN designs that account for the requirements of value cocreation and networked business.

Contribution The study's contribution is twofold. First, extant S-D logic research is dominated by theoretical discourses and lacks factual implications in real-world organizational practices (Day, 2004; Jain et al., 2007; Levy, 2006). This research contributes to $S-D$ logic literature in going one step further in expanding S-D logic beyond the realm of philosophy and theory (see Fig. 1). We employ S-D logic's descriptive knowledge base to derive design requirements and design principles as applicable knowledge to guide the design of DVNs (i.e., prescriptive knowledge). We, thereby, demonstrate the reflection of S-D logic's theoretical basis in organizational practices. Second, our study contributes to value co-creation literature. While existing value co-creation research generically refers to all value co-creation processes, we refer to technologyenabled value co-creation processes aimed at digital service in the context of multi-actor constellations. Extant value cocreation / S-D logic research does underscore networked value co-creation processes, but the role of IT/IS in these processes has remained underserved. Multiple calls for future research in the role of IS/IT in networked value co-creation processes reflect the relevance of pushing further the notion of DVN (Barrett et al., 2015; Böhmann et al., 2014; Breidbach \& Maglio, 2016; Lusch \& Nambisan, 2015).

Implications Researchers are provided with an organizing tripartite structure of interrelated requirements, principles, and features for DVN design to more thoroughly research the intersection of IS and value co-creation (e.g., "technology-enabled value co-creation", Breidbach \& Maglio, 2016). This intersection has repeatedly been emphasized as research priority as follows. While value co-creation research stresses IT and IS as research priorities and, consequently, calls for investigating the roles of IT and IS in value co-creation processes (Akaka \& Vargo, 2014; Alter, 2012; Bitner et al., 2000; Breidbach \& Maglio, 2016; Giebelhausen et al., 2014; Ostrom et al., 2010), IS research calls for revisiting the roles of information, context, environment, service, and customers (Alter \& Browne, 2005; Wand \& Weber, 2002) - all core constructs in the value co-creation / S-D logic perspective. With this study, we pave the way for other researchers to further investigate technology-enabled value co-creation in DVNs. 
Table 7 Design Principles Evaluation in Beta's DVN Design Workshop

\begin{tabular}{|c|c|}
\hline Design Principles & $\begin{array}{l}\text { Beta's DVN Design 1: } \\
\text { Hall Building Platform }\end{array}$ \\
\hline $\begin{array}{l}\text { P1. Principle of } \\
\text { Ecosystem-Oriented } \\
\text { Design }\end{array}$ & $\begin{array}{l}\text { Six dedicated partner roles (service } \\
\text { partners for architecture, hall facades, } \\
\text { maintenance, construction, logistics, } \\
\text { and components) and two dedicated } \\
\text { customer roles (small and medium } \\
\text { enterprises (SMEs), original } \\
\text { equipment manufacturers (OEMs)), } \\
\text { all of which are controlled by Beta's } \\
\text { engagement program that specifies } \\
\text { entrance and exit rules (control } \\
\text { mechanism) }\end{array}$ \\
\hline P2. Principle of & Technology-Oriented Design \\
\hline
\end{tabular}

Design principles guided participants in embracing operan $t$ IT to meet the needs of end customers relative to how competitors apply their operant resources.

P3. Principle of

Mobilization-Oriented Design
Beta's DVN Design 2:

Steel Slug Platform

Four dedicated partner roles (logistics, plant engineering, plant operator, software development) and two dedicated customer roles (SME, OEM), all of which are controlled by Beta's engagement program that specifies entrance and exit rules (control mechanism)

Beta's digital platform (operant IT) for the planning of hall building projects including the integration of hall building activities (i.e., hall planning, product decisions, scheduling, ordering, production, delivery of pre-fabricated and finished materials for assembly)
Online marketplace (transparency mechanism) enlisting Beta's licensed third parties in six roles (architect, facade, maintenance, construction, logistics, components) to identify and mobilize relevant third parties for a given hall building project
Design principles guided participants in embracing transparency mechanisms to identify and mobilize own (internal) and third-party (external) resources.

P4. Principle of Interaction-Oriented Design
Project management feature (protocol of XML-based form for the capture, exchange) accessible to Beta, project-affiliated third parties, and a hall-receiving end customer in a given hall building project with a chat feature (interaction opportunity) for project participants storage, retrieval, and exchange of shaped steel slug orders (protocol of exchange) to organize the interaction between Beta and its customers
Evaluation

Design principles guided participants in embracing specific partner roles and an engagement program to account for DVNs' structural and dynamic complexity.

Custom optimization software (operant IT) for Beta's inhouse laser cutting of shaped steel slugs for several customers on a single steel coil to reduce steel waste

Live tracking feature (transparency mechanism) for Beta's steel slug production managers with an overview of all open steel slug orders by Beta's customers to combine two or more steel slugs with the same material quality and thickness on a single steel coil to reduce steel waste
Design principles guided participants in embracing interaction opportunities and protocols of exchange to engage in mutual value $c o$-creation activities.
Furthermore, this research provides enduring and steering requirements and principles for managers to guide the systematic development of DVNs, which is generally a multi-stage approach that requires repeated checks and 
Table 8 The Relations between Design Requirements, Principles, and Features (with relations in brackets)

Design Requirements (DRs) in Association to

Derivative Propositions (DPs) (Lusch et al., 2007)
Design Principles (Ps) in Association to Design

Requirements (DRs)
Illustrative Design Features (DFs) in Association to Design Principles (Ps)
DR1. DVNs should enhance their participating organizations' competitive advantages by applying operant resources more effectively than non-participating organizations. $(D P I)$

DR2. DVNs should integrate operant resources between participating organizations in designing digital service. $(D P 2)$

DR3. DVNs should embrace information technology as an operan $t$ resource to initiate value co-creation processes. (DP3)

DR4. DVNs should engage customers and third-party actors in value co-creation activities. (DP4)

DR5. DVNs should rely on third-party actors to understand how end customers uniquely integrate resources. (DP5)

DR6. DVNs should provide service $c o$-design opportunities to end customers and third-party actors at once. $(D P 6)$

DR7. DVNs should adopt collaboratively developed, risk-based pricing and cost mechanisms. (DP7)

DR8. DVNs should be orchestrated by a prime resource integrator. $(D P 8)$

DR9. DVNs should treat their participating organizations' employees as operant resources in designing digital service. $(D P 9)$
P1. Principle of Ecosystem-Oriented Design:

Provide the DVN with control mechanisms and specific third-party roles for DVN participants (1) to align the operations of third-party resource integration and (2) to ensure fair sharing of economic risks, costs, and revenues among all DVN participants, given that DVNs are structurally and dynamically complex multi-actor settings.

(DR2, DR7, DR8)

P2. Principle of Technology-Oriented Design: Provide the DVN with operant information technology for DVN participants (1) to exploit data inter-organizationally, (2) to separate informational assets from physical goods, and (3) to facilitate the commercialization of both through digital channels, given that DVN participants are distributed temporally, organizationally, and spatially.

\section{(DR3, DR4)}

P3. Principle of Mobilization-Oriented Design:

Provide the DVN with transparency mechanisms for DVN participants to identify and mobilize own (internal) and third-party (external) resources in innovating and designing digital service, given that DVNs expeditiously mobilize distributed resources for any given end customer context.

(DR1, DR2, DR4, DR9)

P4. Principle of Interaction-Oriented Design:

Provide the DVN with interaction opportunities and a protocol of exchange for DVN participants to engage in mutual value $c o$-creation activities, given that DVNs hold heterogeneous and complementary resources at DVN orchestrators, third party actors, and end customers.

(DR4, DR5, DR6, DR7)
DF1. Extensible Codebase for Enterprise Software:

Semi-open enterprise software serving as a building block upon which third-party derivatives can be added $(P 1, P 2)$

DF2. Enterprise Software Derivatives: Peripheral third-party software, hardware, and services augmenting a core extensible codebase $(P 1, P 4)$

DF3. Integration and Certification Center: Quality management program certifying peripheral third-party derivatives that integrate with a core extensible codebase $(P 2$, P3)

DF4. API Management Software:

Standard enterprise software organizing defined exchange protocols for inter-systems communication $(P 2, P 3)$ rethinking. Adapting to emergent service and networked economies in current business environments is both relevant and complex for managers. Through reflecting the offered requirements and principles, managers can more clearly analyze requirements and design specifications of DVNs that simultaneously adhere to service thinking. This may be especially useful for organizations during early planning and implementation phases of DVNs. Using these principles, managers might anticipate areas of concerns and take appropriate measures in implementing the principles through specific DVN design features.

Limitations Interpreting the design requirements and principles should be done cautiously. First, our EVAL4 remains preliminary due to the nature of the artifact. To conclude that the design principles are useful and effective, more data is required. We provide an intermediate evaluation which shall be picked up and extended by future research.
Vom Brocke et al. (2019) support intermediate contributions in the DSR knowledge accumulation and evaluation process. Moreover, given the socio-technical nature and the scope of the phenomenon of interest, an evaluation comprising real tasks, real systems, and real users (Sonnenberg \& vom Brocke, 2012, p. 396) is resource consuming. While we have adopted a sophisticated iterative research design (see section Research Method), the design principles can be further enhanced through studying different DVN cases. We suggest evaluating the design principles in several instances of running DVNs. Notably, future research is encouraged to iterate the offered requirements and principles in the contexts of different DVN instantiations. Specifically, while we rely on Alpha's DVN success case, failure cases hold fertile ground for advancing the offered requirements and principles. This is because failure cases embody precious case narratives and important learnings, both of which serve as consultable record in identifying effective design knowledge. Moreover, 
prominent IT service providers' DVNs are fruitful environments for such evaluation endeavors in that their sustained DVNs also contain precious design knowledge. Second, while we posit a novel approach for the design of DVNs (i.e., target state), we are lacking an investigation of how existing DVNs adhere to S-D logic / value co-creation's underlying concepts and premises (see section Theoretical Foundation). Third, IS research on design theories distinguishes between design principles that address a system's functionality and methods that addresses a system's development process (Lindgren et al., 2004; Walls et al., 1992). Our research has focused only on DVNs' functionality. Our proposed principles, hence, do not allow for a full-blown, methodological approach to completely designing DVNs from scratch. Rather, we only provide an orientation on what functionality to account for during the iterative and emergent process of developing DVNs. Thus, we see the need for future research on methods that offer methodological guidance in designing DVNs. One application area for such methods is the analysis and design of digital business models (Timmers, 1998; Wieland et al., 2017). In a first attempt of doing so, Alpha has compiled a digital business modelling method. This method leverages the proposed design principles' theoretical base to translate them into a structured method for business modelling. Fourth, in a similar vein, our design requirements and principles are also limited by our chosen S-D logic perspective. Most likely, employing additional perspectives will reveal more relevant design requirements and principles for DVNs.

Conclusion The rise of digital and ecosystemic business leads to new demands in business management. Ecosystems, technology, resource mobilization, and multi-actor interactions play an increasingly pivotal role in such business contexts, which suggests that they are to be placed at the center of new DVNs. This requires principles for guiding the design of such DVNs. Relying on the descriptive insights by S-D logic on the requirements of service business, these design principles deal with the core area of future organizational management such as orchestrating ecosystems, employing operant resources, novel logics of mobilizing resources between actors, and embracing technology-enabled value co-creation.

Acknowledgments This work has been supported by the Swiss National Science Foundation (SNSF).

\section{Appendix 1}

\section{Alpha's DVN}

Alpha has launched a thriving DVN for enterprise software that has grown globally since its launch in 2012. About 13,000 third-party actors complement Alpha's core software package with software extensions (e.g., add-ins, modules, applications), data, consulting services, and sales channels. This thriving ecosystem of third parties (see Fig. 2) is central to Alpha's strategy. In value co-creation processes with Alpha's end customer organizations, Alpha identifies missing functionality in its enterprise software. Thereupon, Alpha mobilizes at least one third-party actor - existing or new - in its DVN to fill this void in functionality. We rely on Alpha's DVN to illustrate the design principles' implementation in an expository DVN case as Alpha that has been effective in (re)forming its thriving DVN for a prolonged period. For more detail on Alpha's DVN, we refer to [blinded for review]. Alpha serves well our purpose of illustrating the design principles in that it operates a service ecosystem that conforms with the defining characteristics of DVNs as outlined next.

First, Alpha's service ecosystem is inextricably intertwined with and facilitated by Alpha's digital platform. Alpha has installed a digital platform for enterprise software that thirdparty actors access via an open platform as a service (PaaS). This platform provides these third-party actors and end customer organizations with technical functionalities and business services for building software extensions, professional services, and sales channels. Alpha's digital platform denotes an entire array of interconnected software products at Alpha, third-party actors, and end customer organizations. This comprehensive array ultimately enables value co-creation processes beyond temporal, organizational, and spatial boundaries.

Second, Alpha's service ecosystem configures value cocreation processes with currently 130 end user organizations. For each end user organization, Alpha dynamically integrates the end user organization's internal resources (e.g., enterprise architecture documentation, extant IS) to devise a digital service that meets the end user organization's requirements. Typically, these end user organizations are multinational enterprises with massive arrangements of interconnected systems and technologies that had been introduced over many years and for different purposes. These organizations opt for Alpha's DVN to obtain finely customized IT solutions through elevated levels of engagement by both Alpha's internal DVN teams and licensed thirdparty consultancies that speak the same language as themselves.

Third, value co-creation processes in Alpha's service ecosystem occur in networked multi-actor settings with licensed third parties (see Fig. 2). These third parties complement the standard software package with industry competence, end-user-specific knowledge, close relationships with end user organizations, reach to end user organizations in each geographical location, and human resources capable of serving as sales force, consultants, and augmenting developers. Alpha orchestrates these different resources contingent on a given end user organization's IT needs. Relying on Alpha's thriving DVN will thus illustrate how the design principles could materialize in Alpha's organizational practices in the form of DVN design features. 


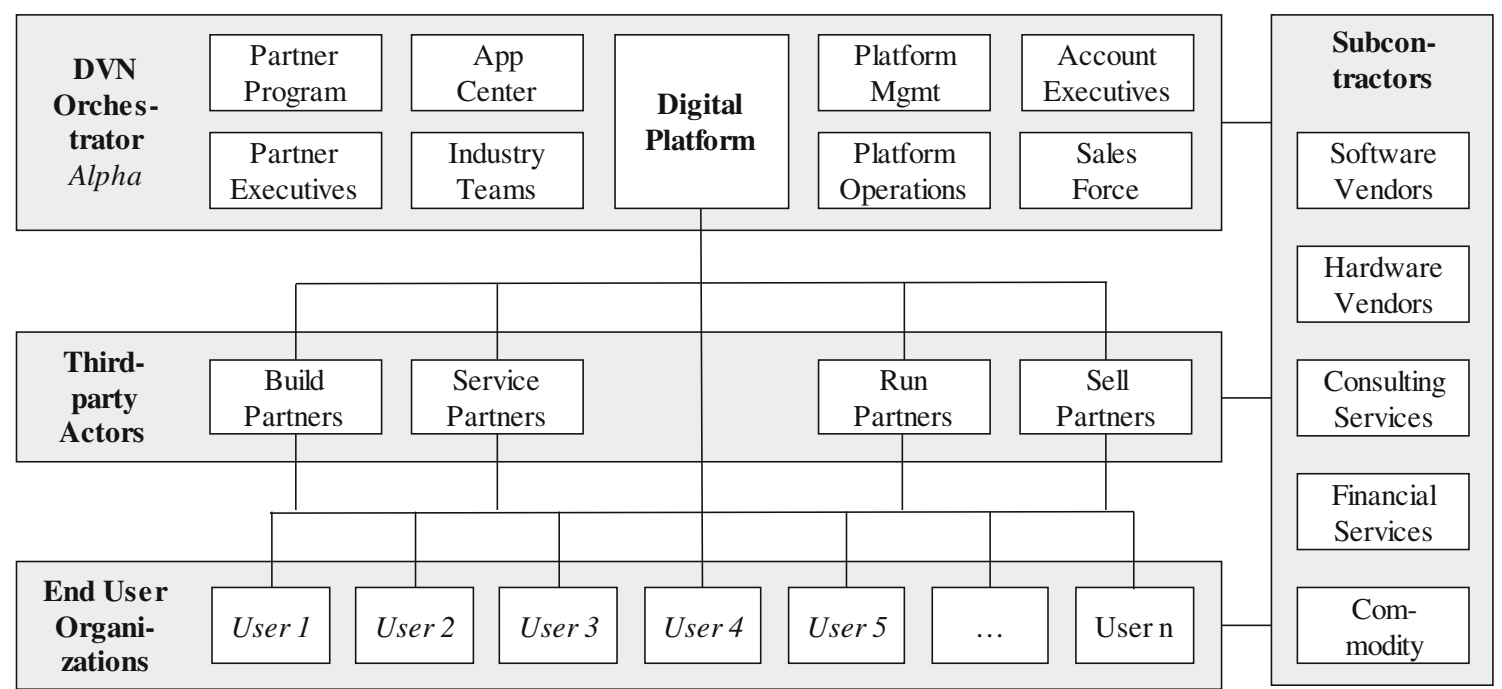

Fig. 2 Overview of Alpha's vigital value co-creation network

\section{Appendix 2}

\section{Beta's DVN design workshop}

Beta's products comprise steel, stainless products, automotive technologies, plant technologies, elevator systems, marine systems, shipbuilding, firearms, and high-speed trains. With 670 subsidiaries worldwide, Beta is one of the world's largest steel producers with about 170,000 employees. To account for the outlined shift toward a service economy, Beta substantially engages in DVN design through value co-creation with its suppliers, third parties, and customers. To ideate two of Beta's DVN ideas, a moderator asked nine Beta employees to work for six-hours in one of two groups on one DVN idea, respectively. First, the moderator distributed an A4 sheet carrying the four design principles (see Table 5) to each participant and drew the participants' attention to two posters also displaying them. Second, the moderator asked the participants to invest $60 \mathrm{~min}$ in reading the design principles to note ideas based on them without interaction. Third, the moderator asked the participants to invest $60 \mathrm{~min}$ in presenting their ideas to the other participants within their group to generate further ideas. Fourth, the two groups were asked to cluster the complete set of ideas, and to reflect these clusters in a network view (see Appendices 7 and 8). The two groups were then asked to analyze how far their DVN designs reflected the features called for by the design principles.

\section{Appendix 3}

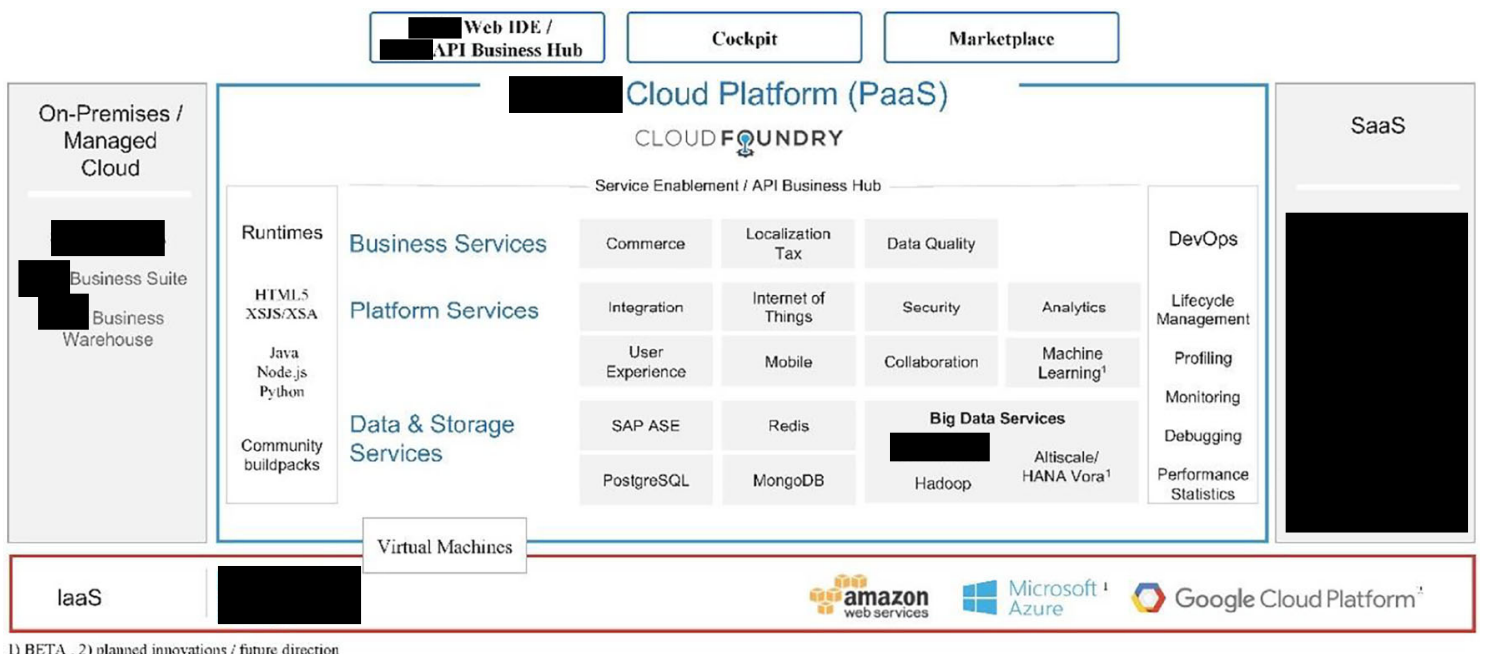

1) BETA , 2) plauned innovations / furure direction

Fig. 3 Extensible codebase of Alpha's DVN (DF1) 


\section{Appendix 4}

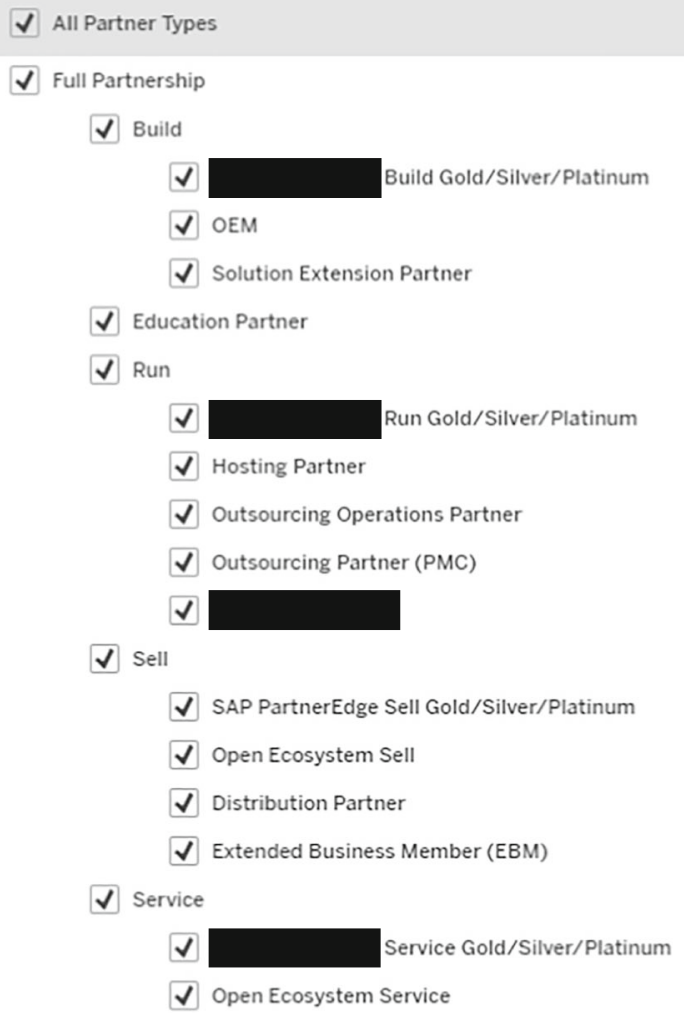

Fig. 4 Enterprise software derivatives of Alpha's DVN (DF2) 


\section{Appendix 5}

Fig. 5 Certified solutions directory of Alpha's DVN (DF3)

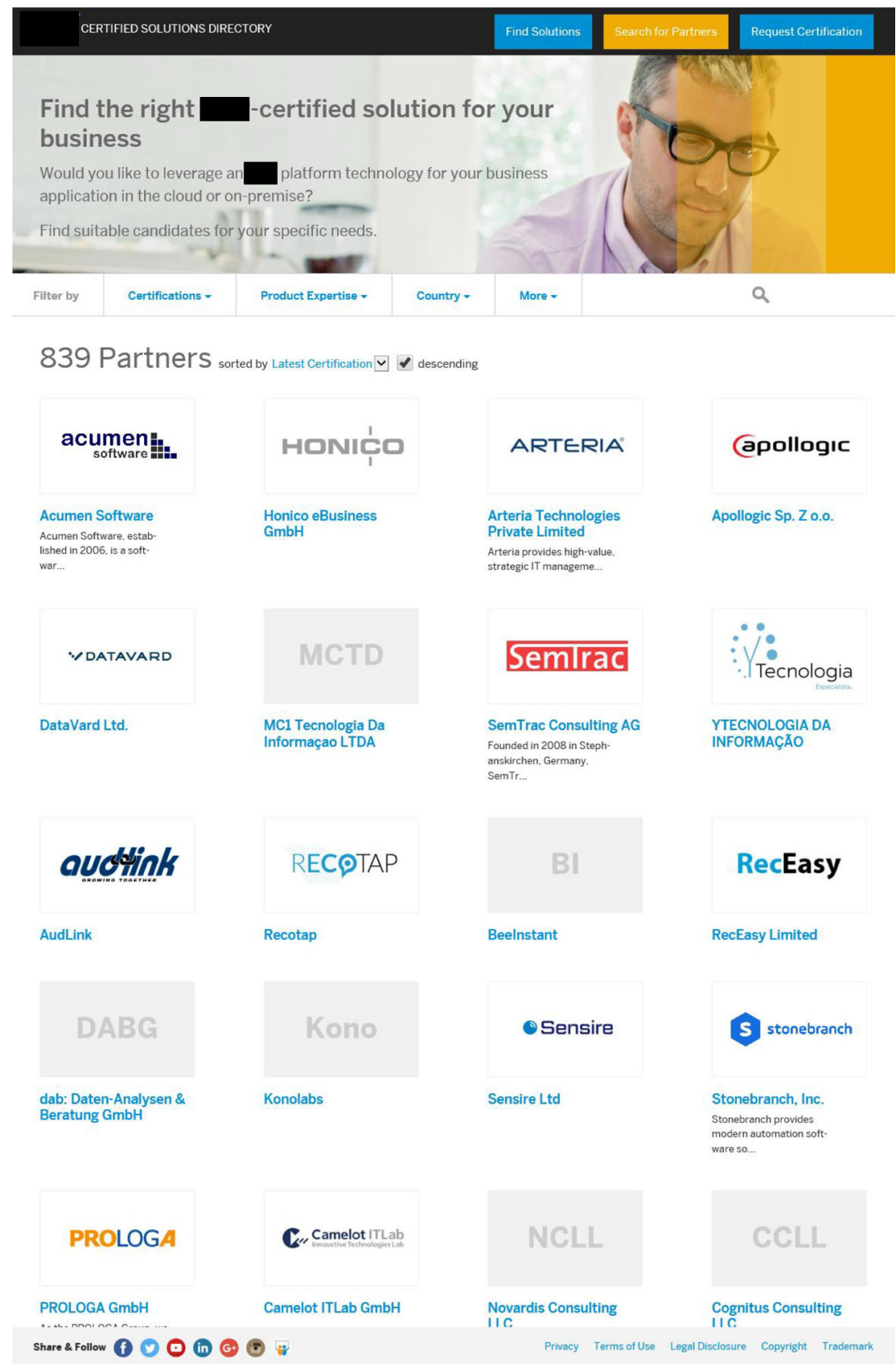




\section{Appendix 6}

\section{ᄋृ}

Cloud Platform API Management

Access APIs seamlessly across heterogenous landscapes using one API platform

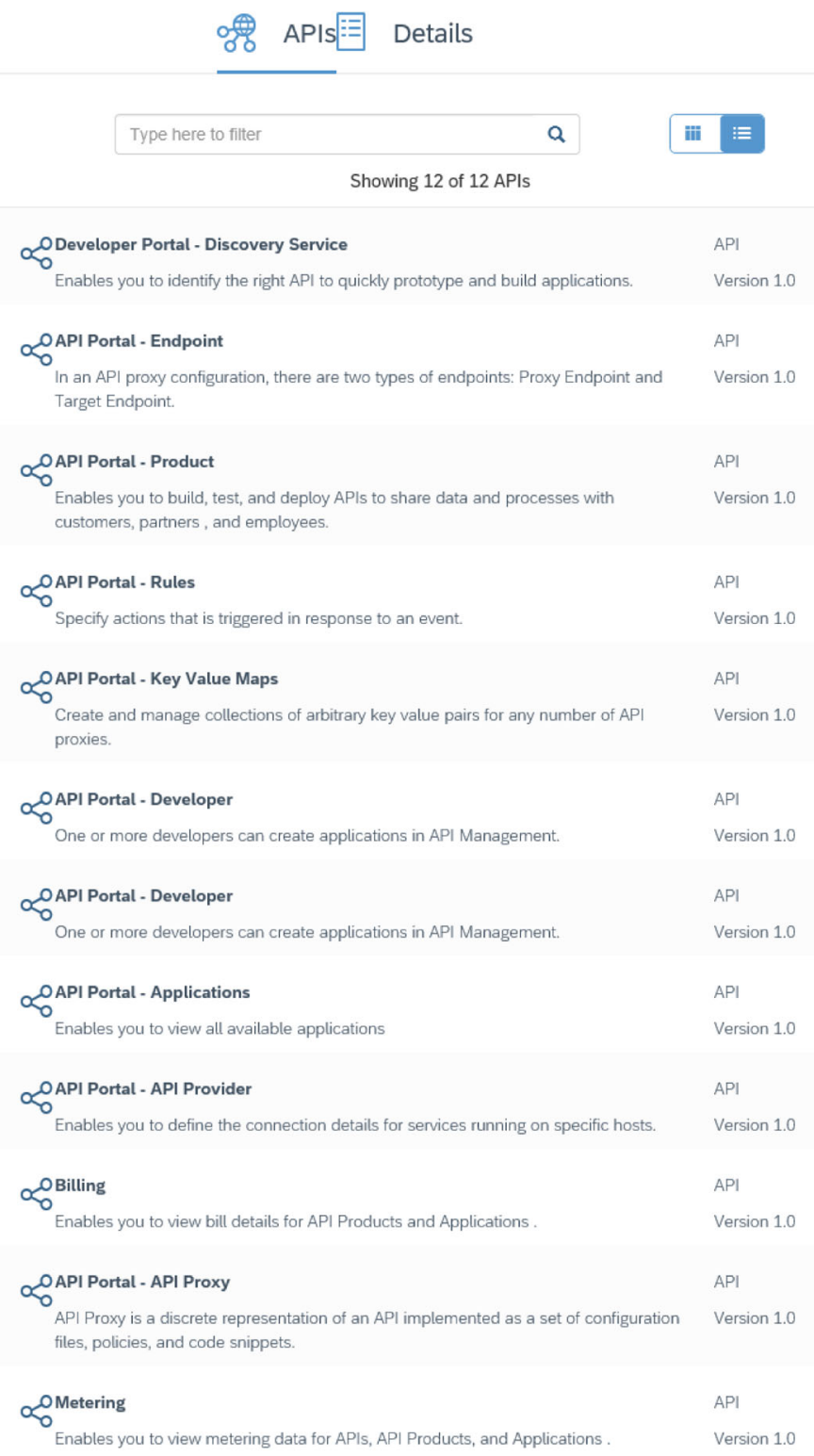

Fig. 6 API management software of Alpha's DVN (DF4) 


\section{Appendix 7}

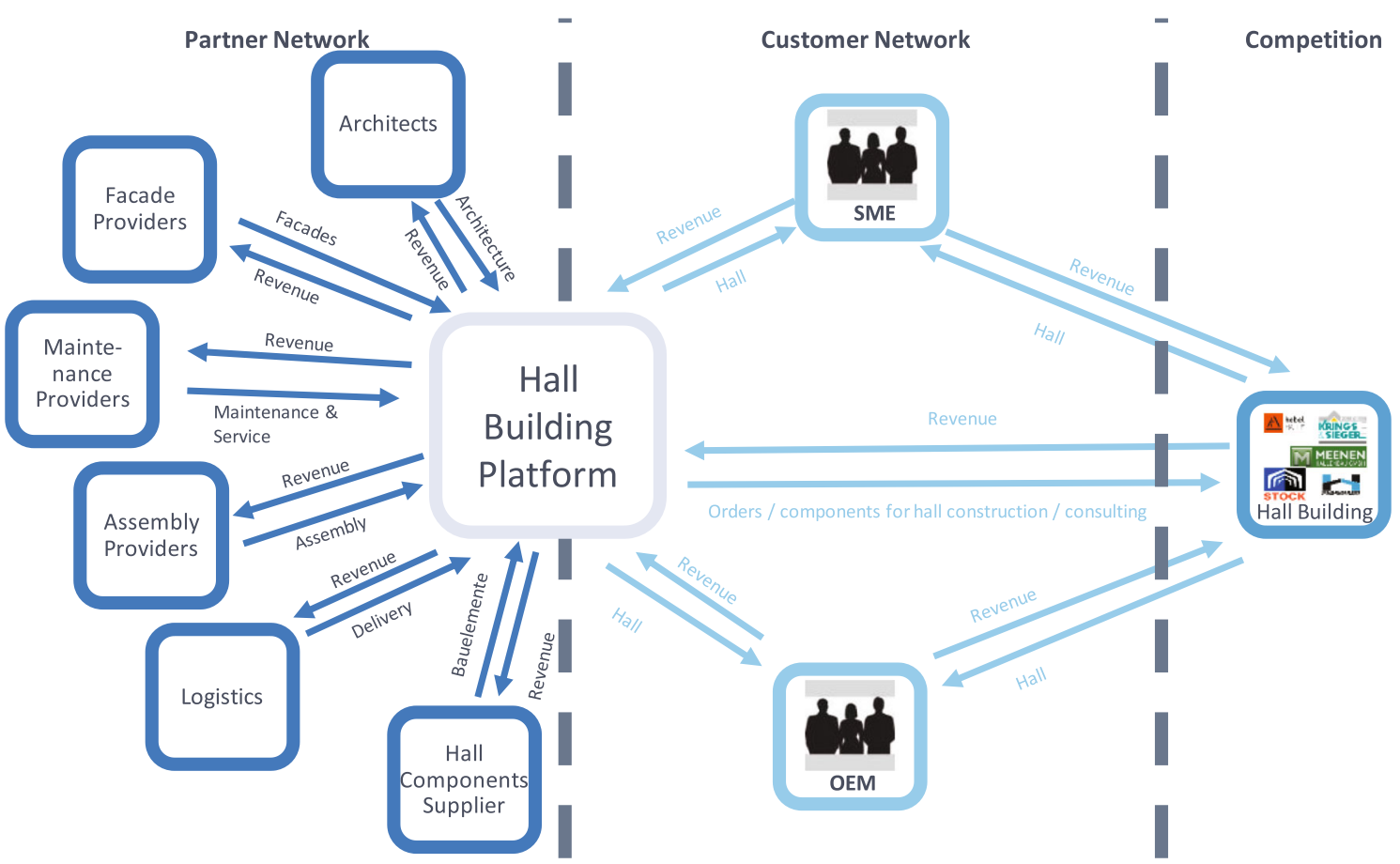

Fig. 7 Beta's DVN Design 1 (Network View): Hall Building Platform. The hall building platform mediates the integration of a hall building project's activities (i.e., hall planning, product decision, scheduling, ordering, production, delivery of pre-fabricated materials for assembly)

\section{Appendix 8}

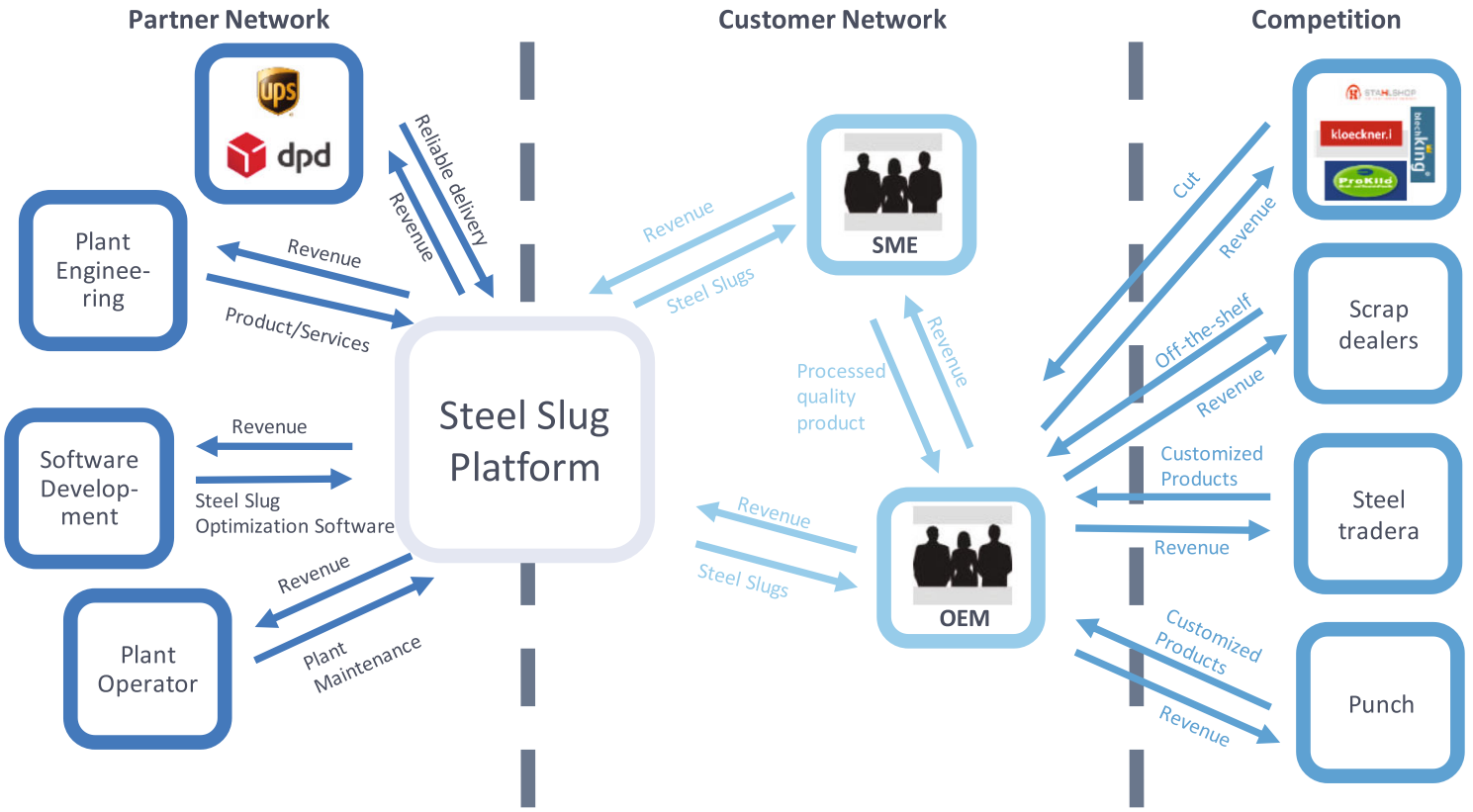

Fig. 8 Beta's DVN Design 2 (Network View): Steel Slug Platform. The steel slug platform mediates Beta's inhouse laser cutting of shaped steel slugs, ordered by different customers, on a single steel coil to reduce steel waste 
Open Access This article is distributed under the terms of the Creative Commons Attribution 4.0 International License (http:// creativecommons.org/licenses/by/4.0/), which permits unrestricted use, distribution, and reproduction in any medium, provided you give appropriate credit to the original author(s) and the source, provide a link to the Creative Commons license, and indicate if changes were made.

\section{References}

Abraham, R., Aier, S., \& Winter, R. (2014). Fail Early, Fail Often: Towards Coherent Feedback Loops in Design Science Research Evaluation. Paper presented at the 2014 International Conference on Information Systems (ICIS 2014), Auckland, New Zealand.

Aier, S., Fischer, C., \& Winter, R. (2011). Construction and Evaluation of a Meta-Model for Enterprise Architecture Design Principles. Paper presented at the The 10th International Conference on Wirtschaftsinformatik WI 2011, Zurich, Switzerland.

Akaka, M. A., \& Vargo, S. L. (2014). Technology as an operant resource in service (eco)systems. Information Systems and e-Business Management, 12(3), 367-384. https://doi.org/10.1007/s10257-0130220-5.

Akaka, M. A., Vargo, S. L., \& Lusch, R. F. (2012). An exploration of networks in value Cocreation: A service-ecosystems view. Review of Marketing Research, 9, 13-50. https://doi.org/10.1108/S15486435(2012)0000009006.

Alt, R. (2016). Electronic markets on customer-orientation. Electronic Markets, 26(3), 195-198. https://doi.org/10.1007/s12525-016$0229-\mathrm{y}$.

Alt, R., \& Zimmermann, H.-D. (2017). Electronic markets on big data services. Electronic Markets, 27(3), 191-195. https://doi.org/10. 1007/s 12525-017-0265-2.

Alter, S. (2012). Challenges for service science. Journal of Information Technology Theory and Application, 13(2), 22.

Alter, S., \& Browne, G. J. (2005). A broad view of systems analysis and design: Implications for research. Communications of the Association for Information Systems, 16(1), 981-999.

Baines, T. (2015). Exploring service innovation and the Servitization of the manufacturing firm. Research Technology Management, 58(5), 9-11. https://doi.org/10.5437/08956308X5805002.

Barile, S., Lusch, R., Reynoso, J., Saviano, M., \& Spohrer, J. (2016). Systems, networks, and ecosystems in service research. Journal of Service Management, 27(4), 652-674. https://doi.org/10.1108/ JOSM-09-2015-0268.

Barrett, M., Davidson, E., Prabhu, J., \& Vargo, S. L. (2015). Service innovation in the digital age: Key contributions and future directions. MIS Quarterly, 39(1), 135-154.

Baskerville, R. L., \& Pries-Heje, J. (2010). Explanatory design theory. Business \& Information Systems Engineering, 2(5), 271-282. https://doi.org/10.1007/s12599-010-0118-4.

Baumol, U., Hollebeek, L., \& Jung, R. (2016). Dynamics of customer interaction on social media platforms. Electronic Markets, 26(3), 199-202. https://doi.org/10.1007/s12525-016-0227-0.

Beirão, G., Patrício, L., \& Fisk, R. P. (2017). Value Cocreation in service ecosystems. Journal of Service Management, 28(2), 227-249. https://doi.org/10.1108/JOSM-11-2015-0357.

Beverungen, D., Müller, O., Matzner, M., Mendling, J., \& Brocke, J. v. (2017). Conceptualizing smart service systems. Electronic Markets, 1-12. https://doi.org/10.1007/s12525-017-0270-5.

Bitner, M. J., Brown, S. W., \& Meuter, M. L. (2000). Technology infusion in service encounters. Journal of the Academy of Marketing Science, 28(1), 138. https://doi.org/10.1177/0092070300281013.
Böhmann, T., Leimeister, J. M., \& Möslein, K. (2014). Service-systemsengineering. Wirtschaftsinformatik, 56(2), 83-90. https://doi.org/10. 1007/s12599-014-0314-8.

Böhmann, T., Leimeister, J. M., \& Möslein, K. (2018). The new Fontiers of service systems engineering. Business \& Information Systems Engineering. https://doi.org/10.1007/s12599-018-0553-1.

Breidbach, C. F., \& Maglio, P. P. (2016). Technology-enabled value cocreation: An empirical analysis of actors, resources, and practices. Industrial Marketing Management, 56, 73-85. https://doi.org/10. 1016/j.indmarman.2016.03.011.

Briscoe, G., Keränen, K., \& Parry, G. (2012). Understanding complex service systems through different lenses: An overview. European Management Journal, 30(5), 418-426. https://doi.org/10.1016/j. emj.2012.06.004.

Brynjolfsson, E., Hu, Y. J., \& Simester, D. (2011). Goodbye Pareto principle, hello long tail: The effect of search costs on the concentration of product sales. Management Science, 57(8), 1373-1386. https:// doi.org/10.1287/mnsc.1110.1371.

Buettner, R. (2017). Getting a job via career-oriented social networking markets. Electronic Markets, 27(4), 371-385. https://doi.org/10. 1007/s12525-017-0248-3.

Ceccagnoli, M., Forman, C., Huang, P., \& Wu, D. J. (2012). Cocreation of value in a platform ecosystem: The case of enterprise software. MIS Quarterly, 36(1), 263-290.

Chandler, J. D., \& Lusch, R. F. (2015). Service systems: A broadened framework and research agenda on value propositions, engagement, and service experience. Journal of Service Research, 18(1), 6-22. https://doi.org/10.1177/1094670514537709.

Chen, J. E., Pan, S. L., \& Ouyang, T. H. (2014). Routine reconfiguration in traditional companies' e-commerce strategy implementation: A trajectory perspective. Information \& Management, 51(2), 270-282. https://doi.org/10.1016/j.im.2013.11.008.

Ciborra, C. (2000). From control to drift: The dynamics of corporate information infrastructures (1st Edition ed.). USA: Oxford University Press.

Constantinides, P., Henfridsson, O., \& Parker, G. G. (2018). Platforms and infrastructures in the digital age. Information Systems Research, 29(2), 381-400. https://doi.org/10.1287/isre.2018.0794.

Davis, M. M., Spohrer, J. C., \& Maglio, P. P. (2011). Guest editorial: How technology is changing the design and delivery of services. Operations Management Research, 4(1-2), 1-5. https://doi.org/10. 1007/s12063-011-0046-6.

Day, G. S. (2004). Achieving advantage with a new dominant logic. Journal of Marketing, 68(1), 18-19.

de Reuver, M., Sørensen, C., \& Basole, R. C. (2018). The digital platform: A research agenda. Journal of Information Technology, 33(2), $124-135$.

Du, K. (2017). Complacency, capabilities, and institutional pressure: Understanding financial institutions' participation in the nascent mobile payments ecosystem. Electronic Markets, 1-13. https://doi. org/10.1007/s12525-017-0267-0.

Eaton, B., Elaluf-Calderwood, S., Sørensen, C., \& Yoo, Y. (2015). Distributed tuning of boundary resources: The case of Apple's Ios service system. MIS Quarterly, 39(1), 217-243.

Galvagno, M., \& Dalli, D. (2014). Theory of value co-creation: A systematic literature review. Managing Service Quality, 24(6), 643683. https://doi.org/10.1108/Msq-09-2013-0187.

Giebelhausen, M., Robinson, S. G., Sirianni, N. J., \& Brady, M. K. (2014). Touch versus tech: When technology functions as a barrier or a benefit to service encounters. Journal of Marketing, 78(4), 113124. https://doi.org/10.1509/jm.13.0056.

Gregor, S., \& Hevner, A. R. (2013). Positioning and presenting design science research for maximum impact. MIS Quarterly, 37(2), 337 355. https://doi.org/10.25300/Misq/2013/37.2.01. 
Grönroos, C. (2011). Value co-creation in service logic: A critical analysis. Marketing Theory, 11(3), 279-301. https://doi.org/10.1177/ 1470593111408177.

Grönroos, C., \& Ravald, A. (2011). Service as business logic: Implications for value creation and marketing. Journal of Service Management, 22(1), 5-22. https://doi.org/10.1108/ 09564231111106893.

Grover, V., \& Kohli, R. (2012). Cocreating it value: New capabilities and metrics for multifirm environments. MIS Quarterly, 36(1), 225-232.

Haki, K., \& Legner, C. (2013). Enterprise Architecture Principles in Research and Practice: Insights from an Exploratory Study. Paper presented at the 21st European Conference on Information Systems (ECIS 2013), Utrecht, Netherlands.

Haki, K., Blaschke, M., Aier, S., \& Winter, R. (2018). A value co-creation perspective on information systems analysis and design. Business \& Information Systems Engineering, Online First. https://doi.org/10. 1007/s12599-018-0557-x.

Hanseth, O., \& Lyytinen, K. (2010). Design theory for dynamic complexity in information infrastructures: The case of building internet. Journal of Information Technology, 25(1), 1-19. https://doi.org/10. 1057/jit.2009.19.

Henfridsson, O., \& Bygstad, B. (2013). The generative mechanisms of digital infrastructure evolution. MIS Quarterly, 37(3), 907-931. https://doi.org/10.25300/Misq/2013/37.3.11.

Hollebeek, L. D. (2013). The customer engagement/value interface: An exploratory investigation. Australasian Marketing Journal (AMJ), 21(1), 17-24. https://doi.org/10.1016/j.ausmj.2012.08.006.

Iivari, J. (2015). Distinguishing and contrasting two strategies for design science research. European Journal of Information Systems, 24(1), 107-115. https://doi.org/10.1057/ejis.2013.35.

Jain, H., Tanniru, M., Spohrer, J., Hsu, C., Zhao, L., \& Zhao, L. (2007). ICIS 2007 panel report: Bridging service computing and service management: How MIS contributes to service orientation? Communications of the Association for Information Systems, 22, 145.

Jeansson, J., Nikou, S., Lundqvist, S., Marcusson, L., Sell, A., \& Walden, P. (2017). SMEs' online channel expansion: Value creating activities. Electronic Markets, 27(1), 49-66. https://doi.org/10.1007/ s12525-016-0234-1.

Kabadayi, S., \& Price, K. (2014). Consumer - Brand engagement on Facebook: Liking and commenting behaviors. Journal of Research in Interactive Marketing, 8(3), 203-223. https://doi.org/10.1108/ JRIM-12-2013-0081.

Karhu, K., Gustafsson, R., \& Lyytinen, K. (2018). Exploiting and defending open digital platforms with boundary resources: Android's five platform forks. Information Systems Research, 29(2), 479-497. https://doi.org/10.1287/isre.2018.0786.

Kazan, E., Tan, C.-W., Lim, E. T. K., Sørensen, C., \& Damsgaard, J. (2018). Disentangling digital platform competition: The case of UK Mobile payment platforms. Journal of Management Information Systems, 35(1), 180-219. https://doi.org/10.1080/ 07421222.2018 .1440772 .

Kruse, L. C., Seidel, S., \& Gregor, S. (2015). Prescriptive Knowledge in IS Research: Conceptualizing Design Principles in Terms of Materiality, Action, and Boundary Conditions. Paper presented at the 48th Hawaii International Conference on System Sciences.

Leminen, S., Westerlund, M., Rajahonka, M., \& Siuruainen, R. (2012). Towards IOT Ecosystems and Business Models. In Andreev S., Balandin S., \& Koucheryavy Y. (eds) Internet of Things, Smart Spaces, and Next Generation Networking (pp. 15-26). ruSMART 2012, NEW2AN 2012. Lecture Notes in Computer Science, vol 7469. Springer, Berlin, Heidelberg.

Levy, S. J. (2006). How new, how dominant? In R. F. Lusch \& S. L. Vargo (Eds.), The service-dominant logic of marketing. Dialog, debate, and directions. New York: M. E. Sharpe.
Lindgren, R., Henfridsson, O., \& Schultze, U. (2004). Design principles for competence management systems: A synthesis of an action research study. MIS Quarterly, 28(3), 435-472.

Loebbecke, C., \& Picot, A. (2015). Reflections on societal and business model transformation arising from digitization and big data analytics: A research agenda. The Journal of Strategic Information Systems, 24(3), 149-157. https://doi.org/10.1016/j.jsis.2015.08.002.

Loukis, E., Janssen, M., Dawes, S., \& Zheng, L. (2016). Evolving ICT and governance in organizational networks - conceptual and theoretical foundations. Electronic Markets, 26(1), 7-14. https://doi.org/ 10.1007/s12525-015-0210-1.

Lusch, R. F., \& Nambisan, S. (2015). Service innovation: A servicedominant logic perspective. MIS Quarterly, 39(1), 155-176.

Lusch, R. F., Vargo, S. L., \& O'Brien, M. (2007). Competing through service: Insights from service-dominant logic. Journal of Retailing, 83(1), 5-18. https://doi.org/10.1016/j.jretai.2006.10.002.

Lusch, R. F., Vargo, S. L., \& Tanniru, M. (2010). Service, value networks and learning. Journal of the Academy of Marketing Science, 38(1), 19-31. https://doi.org/10.1007/s11747-008-0131-z.

Lyytinen, K., Yoo, Y., \& Boland, R. J., Jr. (2016). Digital product innovation within four classes of innovation networks. Information Systems Journal, 26(1), 47-75. https://doi.org/10.1111/isj.12093.

Macdonald, E. K., Kleinaltenkamp, M., \& Wilson, H. N. (2016). How business customers judge solutions: Solution quality and value in use. Journal of Marketing, 80(3), 96-120. https://doi.org/10.1509/ jm.15.0109.

Maglio, P. P., \& Spohrer, J. (2013). A service science perspective on business model innovation. Industrial Marketing Management, 42(5), 665-670. https://doi.org/10.1016/j.indmarman.2013.05.007.

Maglio, P. P., Vargo, S. L., Caswell, N., \& Spohrer, J. (2009). The service system is the basic abstraction of service science. Information Systems and e-Business Management, 7(4), 395-406. https://doi. org/10.1007/s10257-008-0105-1.

Meth, H., Müller, B., \& Mädche, A. (2015). Designing a requirement mining system. Journal of the Association of Information Systems, 16(9), 799-837.

Nambisan, S. (2013). Information technology and product/service innovation: A brief assessment and some suggestions for future research. Journal of the Association for Information Systems, 14 (4), 215 226.

Ngai, E. W. T., Gunasekaran, A., Wamba, S. F., Akter, S., \& Dubey, R. (2017). Big data analytics in electronic markets. Electronic Markets, 27(3), 243-245. https://doi.org/10.1007/s12525-017-0261-6.

Ordanini, A., \& Pasini, P. (2008). Service co-production and value cocreation: The case for a service-oriented architecture (SOA). European Management Journal, 26(5), 289-297.

Ostrom, A. L., Bitner, M. J., Brown, S. W., Burkhard, K. A., Goul, M., Smith-Daniels, V., et al. (2010). Moving forward and making a difference: Research priorities for the science of service. Journal of Service Research, 13(1), 4-36. https://doi.org/10.1177/ 1094670509357611.

Papert, M., \& Pflaum, A. (2017). Development of an ecosystem model for the realization of internet of things (IoT) Services in Supply Chain Management. Electronic Markets, 27(2), 175-189. https:// doi.org/10.1007/s12525-017-0251-8.

Parker, G., Van Alstyne, M., \& Jiang, X. (2017). Platform ecosystems: How developers invert the firm. MIS Quarterly, 41(1), 255-266. https://doi.org/10.25300/Misq/2017/41.1.13.

Payne, A. F., Storbacka, K., \& Frow, P. (2008). Managing the co-creation of value. Journal of the Academy of Marketing Science, 36(1), 8396. https://doi.org/10.1007/s11747-007-0070-0.

Pels, J., \& Vargo, S. L. (2009). Toward a transcending conceptualization of relationship: A service-dominant logic perspective. Journal of Business \& Industrial Marketing, 24, 373-379.

Perks, H., Gruber, T., \& Edvardsson, B. (2012). Co-creation in radical service innovation: A systematic analysis of microlevel processes. 
Journal of Product Innovation Management, 29(6), 935-951. https://doi.org/10.1111/j.1540-5885.2012.00971.x.

Prahalad, C. K., \& Ramaswamy, V. (2004). Co-creating unique value with customers. Strategy \& Leadership, 32(3), 4-9.

Ranjan, K. R., \& Read, S. (2016). Value co-creation: Concept and measurement. Journal of the Academy of Marketing Science, 44(3), 290-315. https://doi.org/10.1007/s11747-014-0397-2.

Richardson, G. L., Jackson, B. M., \& Dickson, G. W. (1990). A principles-based Enterprise architecture: Lessons from Texaco and star Enterprise. MIS Quarterly, 14(4), 385-403. https://doi.org/10. 2307/249787.

Ross, J., Sebastian, I., Beath, C., Mocker, M., Moloney, K., \& Fonstad, N. (2016). Designing and Executing Digital Strategies. Paper presented at the The International Conference on Information Systems, ICIS 2016.

Sarker, S., Sarker, S., Sahaym, A., \& Bjorn-Andersen, N. (2012). Exploring value cocreation in relationships between an ERP vendor and its partners: A revelatory case study. MIS Quarterly, 36(1), 317338

Sonnenberg, C., \& vom Brocke, J. (2012). Evaluations in the Science of the Artificial - Reconsidering the Build-Evaluate Pattern in Design Science Research. Paper presented at the 7th International Conference on Design Science Research in Information Systems and Technology (DESRIST 2012), Las Vegas, USA

Srivastava, S. C., \& Shainesh, G. (2015). Bridging the service divide through digitally enabled service innovations: Evidence from Indian healthcare service providers. MIS Quarterly, 39(1), 245-267.

Stickdorn, M., \& Schwarzenberger, K. (2016). Service design in tourism. Entrepreneurship und Tourismus: Unternehmerisches Denken und Erfolgskonzepte aus der Praxis, 2261.

Tan, B., Pan, S. L., Xianghua, L., \& Lihua, H. (2015). The role of IS capabilities in the development of multi-sided platforms: The digital ecosystem strategy of Alibaba.com. Journal of the Association for Information Systems, 16(4), 248-280.

Tilson, D., Lyytinen, K., \& Sørensen, C. (2010). Digital infrastructures: The missing IS research agenda. Information Systems Research, 21(4), 748-759.

Timmers, P. (1998). Business models for electronic markets. Electronic Markets, 8(2), 3-8.

Tiwana, A. (2015). Platform desertion by app developers. Journal of Management Information Systems, 32(4), 40-77. https://doi.org/ 10.1080/07421222.2015.1138365

Vargo, S. L., \& Lusch, R. F. (2004). Evolving to a new dominant logic for marketing. Journal of Marketing, 68(1), 1-17. https://doi.org/10. 1509/jmkg.68.1.1.24036.

Vargo, S. L., \& Lusch, R. F. (2008). Service-dominant logic: Continuing the evolution. Journal of the Academy of Marketing Science, 36(1), 1-10. https://doi.org/10.1007/s11747-007-0069-6.

Vargo, S. L., \& Lusch, R. F. (2011). It's all B2B... and beyond: Toward a systems perspective of the market. Industrial Marketing Management, 40(2), 181-187. https://doi.org/10.1016/j. indmarman.2010.06.026
Vargo, S. L., \& Lusch, R. F. (2016). Institutions and axioms: An extension and update of service-dominant logic. Journal of the Academy of Marketing Science, 44(1), 5-23. https://doi.org/10.1007/s11747015-0456-3.

Vargo, S. L., \& Lusch, R. F. (2017). Service-dominant logic 2025. International Journal of Research in Marketing, 34(1), 46-67. https://doi.org/10.1016/j.jiresmar.2016.11.001.

Vargo, S. L., Lusch, R. F., \& Akaka, M. A. (2010). Advancing service science with service-dominant logic. In P. P. Maglio, C. A. Kieliszewski, \& J. C. Spohrer (Eds.), Handbook of service science (pp. 133-156). Boston: Springer US.

Venable, J., Pries-Heje, J., \& Baskerville, R. (2014). FEDS: A framework for evaluation in design science research. European Journal of Information Systems, 25(1), 77-89. https://doi.org/10.1057/ejis. 2014.36.

vom Brocke, J., Fay, M., Schmiedel, T., Petry, M., Krause, F., \& Teinzer, T. (2017). A Journey of Digital Innovation and Transformation: The Case of Hilti. In G. Oswald \& M. Kleinemeier (Eds.), Shaping the Digital Enterprise (pp. 237-251).

vom Brocke, J., Winter, R., Hevner, A.R. \& Maedche, A. (2019). Accumulation and evolution of design knowledge in design science research: A journey through time and space. Journal of the Association for Information Systems, online first.

Walls, J. G., Widmeyer, G. R., \& El Sawy, O. A. (1992). Building an information system design theory for vigilant EIS. Information Systems Research, 3(1), 36-59. https://doi.org/10.1287/isre.3.1.36.

Wand, Y., \& Weber, R. (2002). Research commentary: Information systems and conceptual modeling - a research agenda. Information Systems Research, 13(4), 363-376. https://doi.org/10.1287/isre.13. 4.363.69.

Wieland, H., Hartmann, N. N., \& Vargo, S. L. (2017). Business models as service strategy. Journal of the Academy of Marketing Science, 45(6), 1-19. https://doi.org/10.1007/s11747-017-0531-z.

Wieneke, A., \& Lehrer, C. (2016). Generating and exploiting customer insights from social media data. Electronic Markets, 26(3), 245268. https://doi.org/10.1007/s12525-016-0226-1.

Wirtz, B. W., Pistoia, A., Ullrich, S., \& Göttel, V. (2016). Business models: Origin, development and future research perspectives. Long Range Planning, 49(1), 36-54. https://doi.org/10.1016/j.lrp. 2015.04.001.

Yan, J. Q., Ye, K., Wang, H. Q., \& Hua, Z. S. (2010). Ontology of collaborative manufacturing: Alignment of service-oriented framework with service-dominant logic. Expert Systems with Applications, 37(3), 2222-2231

Zott, C., \& Amit, R. (2008). The fit between product market strategy and business model: Implications for firm performance. Strategic Management Journal, 29(1), 1-26. https://doi.org/10.1002/smj.642.

Publisher's note Springer Nature remains neutral with regard to jurisdictional claims in published maps and institutional affiliations. 